\title{
DIHEDRAL GAUSS HYPERGEOMETRIC FUNCTIONS
}

\author{
Raimundas VIDUNAS \\ (Received 12 August 2010 and revised 2 December 2010)
}

\begin{abstract}
Gauss hypergeometric functions with a dihedral monodromy group can be expressed as elementary functions, since their hypergeometric equations can be transformed to Fuchsian equations with cyclic monodromy groups by a quadratic change of the argument variable. This paper presents general elementary expressions of these dihedral hypergeometric functions, involving finite bivariate sums expressible as terminating Appell's $F_{2}$ or $F_{3}$ series. Additionally, trigonometric expressions for the dihedral functions are presented, and degenerate cases (logarithmic, or with the monodromy group $\mathbb{Z} / 2 \mathbb{Z}$ ) are considered.
\end{abstract}

\section{Introduction}

As is well known, special cases of the Gauss hypergeometric function ${ }_{2} \mathrm{~F}_{1}\left(\begin{array}{c}A, B \\ C\end{array} \mid z\right)$ can be represented in terms of elementary functions. A particularly interesting case are hypergeometric functions with a dihedral monodromy group; they can be expressed with square roots inside power or logarithmic functions. The simplest examples are

$$
\begin{aligned}
& { }_{2} \mathrm{~F}_{1}\left(\begin{array}{c|c}
\frac{a}{2}, \frac{a+1}{2} & z \\
a+1 & z
\end{array}\right)=\left(\frac{1+\sqrt{1-z}}{2}\right)^{-a}, \\
& { }_{2} \mathrm{~F}_{1}\left(\begin{array}{c|c}
\frac{a}{2}, & \frac{a+1}{2} \\
\frac{1}{2} & z
\end{array}\right)=\frac{(1-\sqrt{z})^{-a}+(1+\sqrt{z})^{-a}}{2}, \\
& { }_{2} \mathrm{~F}_{1}\left(\begin{array}{c|c}
\frac{a+1}{2}, \frac{a+2}{2} & z \\
\frac{3}{2} & z
\end{array}\right)=\frac{(1-\sqrt{z})^{-a}-(1+\sqrt{z})^{-a}}{2 a \sqrt{z}} \quad(a \neq 0), \\
& { }_{2} \mathrm{~F}_{1}\left(\begin{array}{c|c}
\frac{1}{2}, & 1 \\
\frac{3}{2} & z
\end{array}\right)=\frac{\log (1+\sqrt{z})-\log (1-\sqrt{z})}{2 \sqrt{z}}=\frac{\arctan \sqrt{-z}}{\sqrt{-z}}, \\
& { }_{2} \mathrm{~F}_{1}\left(\begin{array}{c|c}
\frac{1}{2} & \frac{1}{2} \\
\frac{3}{2} & z
\end{array}\right)=\frac{\log (\sqrt{1-z}+\sqrt{-z})}{\sqrt{-z}}=\frac{\arcsin \sqrt{z}}{\sqrt{z}} .
\end{aligned}
$$

These are solutions of the hypergeometric differential equation with the local exponent differences $1 / 2,1 / 2, a$ at the three singular points. The monodromy group is an infinite

2000 Mathematics Subject Classification: Primary 33C05; Secondary 33C65, 68W30.

Keywords: Gauss hypergeometric function; monodromy group; dihedral group; Liouvillian solution.

(C) 2011 Faculty of Mathematics, Kyushu University 
dihedral group (for general $a \in \mathbb{C}$ ), or a finite dihedral group (for rational non-integer $a$ ), or an order 2 group (for non-zero integers $a$ ). We refer to Gauss hypergeometric functions with a dihedral monodromy group as dihedral hypergeometric functions.

Despite a rich history of research of Gauss hypergeometric functions, only the simplest formulas for dihedral ${ }_{2} \mathrm{~F}_{1}$ functions like above are given in the common literature, say $[\mathbf{1}, 15.1]$ and $[4,2.8]$. General dihedral ${ }_{2} \mathrm{~F}_{1}$ functions are contiguous to the simplest functions given above (or their fractional-linear transformations); that is, their upper and lower parameters differ by integers from the respective parameters of the simplest functions. The local exponent differences of their hypergeometric equations are $k+1 / 2, \ell+1 / 2$ and $\lambda \in \mathbb{C}$, where $k, \ell$ are integers.

An interesting problem is to find explicit elementary expressions for general dihedral Gauss hypergeometric functions. A set of these expressions is presented in Sections 3, 4 and 6 of this paper. The most general canonical expressions are given in Section 3, in terms of terminating Appell's $F_{2}$ double sums. The key observation is that a particular univariate specialization of Appell's $F_{2}$ function satisfies the same Fuchsian equation as a quadratic transformation of general hypergeometric equations; see Theorem 2.1. When the monodromy group of the hypergeometric equation is dihedral, the alluded $F_{2}$ function is a terminating double sum. Linear relations between dihedral ${ }_{2} \mathrm{~F}_{1}$ and terminating $F_{2}$ solutions of the same Fuchsian equation give the announced elementary expressions for the former.

Section 4 looks at the simpler case of dihedral hypergeometric equations with the local monodromy differences $k+1 / 2,1 / 2, \lambda$. Then the terminating $F_{2}$ double sums become terminating ${ }_{2} \mathrm{~F}_{1}$ sums. The case of the local exponent differences $k+1 / 2, k+1 / 2, \lambda$ can be reduced to the described case by a quadratic transformation of the hypergeometric equation.

Section 5 considers comprehensively degenerate cases with $\lambda \in \mathbb{Z}$.

Section 6 considers trigonometric formulas for dihedral Gauss hypergeometric functions. The simplest such expressions are (see $[\mathbf{1}, 15.1]$ and $[4,2.8])$

$$
\begin{aligned}
& { }_{2} \mathrm{~F}_{1}\left(\begin{array}{c}
\frac{a}{2},-\frac{a}{2} \\
\frac{1}{2}
\end{array} \mid \sin ^{2} x\right)=\cos a x, \\
& { }_{2} \mathrm{~F}_{1}\left(\begin{array}{c}
\frac{1+a}{2}, \frac{1-a}{2} \mid \\
\frac{3}{2}
\end{array} \mid \sin ^{2} x\right)=\frac{\sin a x}{a \sin x} .
\end{aligned}
$$

The supplementary paper [8] presents similar expressions (as double hypergeometric sums) for quadratic invariants for hypergeometric equations with the dihedral monodromy group, and describes rational pull-back transformations between dihedral hypergeometric functions.

\section{Preliminary facts}

Basic facts on hypergeometric functions, Fuchsian equations, the monodromy group, contiguous relations, terminating hypergeometric sums and Zeilberger's algorithm are well known. We suggest $[\mathbf{2}, \mathbf{3}, \mathbf{5}]$ as standard though overwhelming references. Wikipedia pages $[\mathbf{1 0}]$ can be satisfactorily consulted for quick references. 


\subsection{The hypergeometric equation}

The Gauss hypergeometric function ${ }_{2} \mathrm{~F}_{1}\left({ }_{C}^{A, B} \mid z\right)$ satisfies the hypergeometric differential equation [2, Formula (2.3.5)]

$$
z(1-z) \frac{d^{2} y(z)}{d z^{2}}+(C-(A+B+1) z) \frac{d y(z)}{d z}-A B y(z)=0 .
$$

This is a canonical Fuchsian equation on $\mathbb{P}^{1}$ with three singular points. The singularities are $z=0,1, \infty$, and the local exponents are

$$
0,1-C \text { at } z=0, \quad 0, C-A-B \text { at } z=1 \quad \text { and } \quad A, B \text { at } z=\infty .
$$

The local exponent differences at the singular points are equal (up to a sign) to $1-C$, $C-A-B$ and $A-B$, respectively. Let us denote a hypergeometric equation with the local exponent differences $d_{1}, d_{2}, d_{3}$ by $E\left(d_{1}, d_{2}, d_{3}\right)$, and consider the order of the three arguments unimportant.

Because of frequent use, we recall Euler's and Pfaff's fractional-linear transformations [2, Theorem 2.2.5]:

$$
\begin{aligned}
{ }_{2} \mathrm{~F}_{1}\left(\begin{array}{c|c}
a, b & z \\
c & =
\end{array}\right) \\
=(1-z)^{c-a-b}{ }_{2} \mathrm{~F}_{1}\left(\begin{array}{c}
c-a, c-b \\
c
\end{array} \mid z\right) \\
\mathrm{F}_{1}\left(\begin{array}{c|c}
a, c-b & \frac{z}{z-1}
\end{array}\right) .
\end{aligned}
$$

The following quadratic transformation [2, (3.1.3), (3.1.9), (3.1.7)] will illustrate the key reduction of the dihedral monodromy group to a cyclic monodromy group:

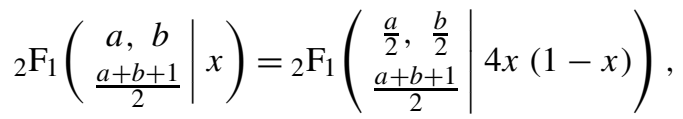

$$
\begin{aligned}
& { }_{2} \mathrm{~F}_{1}\left(\begin{array}{c}
a, b \\
a-b+1
\end{array} \mid x\right)=(1+x)^{-a}{ }_{2} \mathrm{~F}_{1}\left(\begin{array}{c|c}
\frac{a}{2}, \frac{a+1}{2} & \frac{4 x}{a-b+1} \\
a-x)^{2}
\end{array}\right), \\
& { }_{2} \mathrm{~F}_{1}\left(\begin{array}{c|c}
a, b & x \\
2 b & x
\end{array}\right)=\left(1-\frac{x}{2}\right)^{-a}{ }_{2} \mathrm{~F}_{1}\left(\begin{array}{c}
\frac{a}{2}, \frac{a+1}{2} \\
b+\frac{1}{2}
\end{array} \mid \frac{x^{2}}{(2-x)^{2}}\right) .
\end{aligned}
$$

The hypergeometric equations are related in the same way, by a quadratic pull-back transformation of the form

$$
z \longmapsto \varphi(x), \quad y(z) \longmapsto Y(x)=\theta(x) y(\varphi(x)),
$$

where $\varphi(x)$ is a rational (quadratic in this case) function, and $\theta(x)$ is a power factor. Geometrically, the transformation pull-backs the starting differential equation on the projective line $\mathbb{P}_{z}^{1}$ to a differential equation on the projective line $\mathbb{P}_{x}^{1}$, with respect to the covering $\varphi: \mathbb{P}_{x}^{1} \rightarrow \mathbb{P}_{z}^{1}$. The factor $\theta(x)$ shifts the local exponents of the pull-backed equation, but it does not change the local exponent differences. We write the quadratic transformation of hypergeometric equations as

$$
E\left(\frac{1}{2}, \lambda, \mu\right) \stackrel{2}{\leftarrow} E(\lambda, \lambda, 2 \mu),
$$

as the local exponent differences $1 / 2, \mu$ are doubled by the quadratic substitution, and the point with the local exponent difference $1 / 2$ becomes non-singular after an appropriate choice of $\theta(x)$. The arrow follows the direction of the pull-back covering. 


\subsection{The monodromy group}

The simplest hypergeometric equations with a dihedral monodromy group are $E(1 / 2,1 / 2, a)$. The monodromy representation for these equations group can be computed using explicit expressions (1.1)-(1.4). If $a \neq 0$, we take (1.2) and $a \sqrt{z}$ times (1.3) as a basis of solutions; analytic continuation along loops around $z=0$ and $z=1$ gives the following generators of the monodromy group:

$$
\left(\begin{array}{rr}
1 & 0 \\
0 & -1
\end{array}\right) \quad \text { and }\left(\begin{array}{cc}
\frac{1+w}{2} & \frac{1-w}{2} \\
\frac{1-w}{2} & \frac{1+w}{2}
\end{array}\right), \quad w=\exp (-2 \pi i a) .
$$

If $a=0$, the monodromy generators are $\left(\begin{array}{cc}1 & 0 \\ 0 & -1\end{array}\right)$ and $\left(\begin{array}{ll}1 & 2 \pi i \\ 0 & 1\end{array}\right)$.

In general, dihedral hypergeometric functions are contiguous to $E(1 / 2,1 / 2, a)$. They are characterized by the property that their differences of local exponents at two of the three singular points are half-integers. If the third local exponent is an irrational number, the monodromy group is an infinite dihedral group; if it is a non-integer rational number, the monodromy group is a finite dihedral group. If the third local exponent difference is an integer, the monodromy group is isomorphic either to $\mathbb{Z} / 2 \mathbb{Z}$ or (in the presence of logarithmic solutions) to an infinite dihedral group. The distinction of these two cases is given in Theorem 5.1.

The main results of this paper are stated for solutions of the hypergeometric equation (2.1) with

$$
A=\frac{a}{2}, \quad B=\frac{a+1}{2}+\ell, \quad C=\frac{1}{2}-k .
$$

The local exponent differences of our working hypergeometric equation are $k+1 / 2, \ell+1 / 2$ and $\lambda=a+k+\ell$. Throughout this paper, $k, \ell, m$ denote non-negative integers. Except in Section 5, we assume that $a$ is not an integer.

\subsection{The main observation}

If we apply a quadratic pull-back transformation to a hypergeometric equation, and the two ramified points are singularities of the equation, the transformed equation is a Fuchsian equation with generally four singular points (and can be solved in terms of Heun functions). Suppose that the hypergeometric equation has a dihedral monodromy group, hence it is $E\left(k+\frac{1}{2}, \ell+\frac{1}{2}, \lambda\right)$, and the two ramified points are the points with the half-integer local exponent differences. Then the local exponent differences of the transformed equation are $2 k+1,2 \ell+1, \lambda, \lambda$. With an appropriate choice of the power factor $\theta(x)$ in (2.7), the transformed equation has trivial monodromy around the two points with the integer local exponent differences $2 k+1,2 \ell+1$. These two points are not logarithmic because the corresponding points with half-integer local exponent differences are not logarithmic. The monodromy action for the transformed equation will come only from the other two points. The global monodromy group is therefore cyclic, and the monodromy representation is reducible.

In the simplest case $k=0, \ell=0$ the transformed equation has just two singularities. Correspondingly, the classical quadratic transformation (2.4) gives

$$
{ }_{2} \mathrm{~F}_{1}\left(\begin{array}{c}
\frac{a}{2}, \frac{a+1}{2} \\
a+1
\end{array} \mid 4 x(1-x)\right)={ }_{2} \mathrm{~F}_{1}\left(\begin{array}{c}
a, a+1 \\
a+1
\end{array} \mid x\right)=(1-x)^{-a} .
$$


This formula is equivalent to (1.1). If exactly one of $k, \ell$ is zero, the transformed equation is equivalent to a hypergeometric equation again. The transformation is then

$$
E\left(\frac{1}{2}, k+\frac{1}{2}, \lambda\right) \stackrel{2}{\leftarrow} E(2 k+1, \lambda, \lambda),
$$

and the transformed solutions can be expressed in terms of terminating ${ }_{2} \mathrm{~F}_{1}$ sums, as we demonstrate in Section 4.

Even with general integer $k, \ell$, the transformed solution must have elementary power (at worst logarithmic) solutions, because of the reducible monodromy group. With a proper normalization by $\theta(x)$ in (2.7) the elementary power solutions can be polynomials in $x$. It turns out that those polynomials can be written as terminating Appell's $F_{2}$ or $F_{3}$ hypergeometric sums. Recall that Appell's $F_{2}$ and $F_{3}$ bivariate functions are defined by the series

$$
\begin{gathered}
F_{2}\left(\begin{array}{c}
a ; b_{1}, b_{2} \\
c_{1}, c_{2}
\end{array} \mid x, y\right)=\sum_{p=0}^{\infty} \sum_{q=0}^{\infty} \frac{(a)_{p+q}\left(b_{1}\right)_{p}\left(b_{2}\right)_{q}}{\left(c_{1}\right)_{p}\left(c_{2}\right)_{q} p ! q !} x^{p} y^{q}, \\
F_{3}\left(\begin{array}{c}
a_{1}, a_{2} ; b_{1}, b_{2} \\
c
\end{array} \mid x, y\right)=\sum_{i=0}^{\infty} \sum_{j=0}^{\infty} \frac{\left(a_{1}\right)_{p}\left(a_{2}\right)_{q}\left(b_{1}\right)_{p}\left(b_{2}\right)_{q}}{(c)_{p+q} p ! q !} x^{p} y^{q} .
\end{gathered}
$$

As usual, $(a)_{n}$ denotes the Pochhammer symbol (also called raising factorial), which is the product $a(a+1) \cdots(a+n-1)$.

The key fact is that quadratic transformations of general hypergeometric equations coincide with the differential equations for some univariate specializations of Appell's $F_{2}$ or $F_{3}$ functions. In particular, the following theorem is proved in [9].

THEOREM 2.1. The functions

$$
F_{2}\left(\begin{array}{c}
a ; b_{1}, b_{2} \\
2 b_{1}, 2 b_{2}
\end{array} \mid x, 2-x\right) \quad \text { and } \quad(x-2)^{-a}{ }_{2} \mathrm{~F}_{1}\left(\begin{array}{c|c}
\frac{a}{2}, \frac{a+1}{2}-b_{2} & \frac{x^{2}}{(2-x)^{2}} \\
b_{1}+\frac{1}{2} & { }^{2}
\end{array}\right.
$$

satisfy the same second-order Fuchsian equation.

Proof. Part 1 of Theorem 2.4 in [9].

For general values of the three parameters $a, b_{1}, b_{2}$, the double series for the $F_{2}(x$, $2-x$ ) function does not converge for any $x$. However, when $b_{1}$ and $b_{2}$ are zero or negative integers, the $F_{2}(x, 2-x)$ function can be seen as a finite sum of $\left(1-b_{1}\right)\left(1-b_{2}\right)$ terms; see Remark 2.3. On the other hand, the ${ }_{2} \mathrm{~F}_{1}$ function in (2.15) is contiguous to the ${ }_{2} \mathrm{~F}_{1}$ function in (1.2) for integer values of $b_{1}, b_{2}$, hence in general it has a dihedral monodromy group as well. This relation between terminating $F_{2}(x, 2-x)$ sums and dihedral hypergeometric functions is behind our explicit expressions for general dihedral functions. We formulate the following variation of Theorem 2.1.

COROLlaRY 2.2. For non-positive integers $k$, $\ell$, the functions

$$
{ }_{2} \mathrm{~F}_{1}\left(\begin{array}{c|c}
\frac{a}{2}, \frac{a+1}{2}+\ell & z \\
\frac{1}{2}-k & z
\end{array}\right) \text { and }(1+\sqrt{z})^{-a} F_{2}\left(\begin{array}{c}
a ;-k,-\ell \\
-2 k,-2 \ell
\end{array} \mid \frac{2 \sqrt{z}}{1+\sqrt{z}}, \frac{2}{1+\sqrt{z}}\right)
$$

satisfy the same second-order Fuchsian equation. 
Proof. Substitute $b_{1}=-k, b_{2}=-\ell$ and $x=2 \sqrt{z} /(1+\sqrt{z})$ in Theorem 2.1.

We present explicit consequences of this coincidence of differential equations in Section 3. Note that if $k=0$ or $\ell=0$ then the double $F_{2}(x, 2-x)$ sum becomes a terminating ${ }_{2} \mathrm{~F}_{1}(x)$ sum, in agreement with an observation after formula (2.11).

Appell's $F_{2}(x, y)$ and $F_{3}(x, y)$ functions are closely related. They satisfy the same system of partial differential equations up to a simple transformation, and particularly, terminating $F_{2}$ sums become terminating $F_{3}$ sums when summation is reversed in both directions. In particular, for $(a)_{k+\ell} \neq 0$ we have

$$
F_{2}\left(\begin{array}{c}
a ;-k,-\ell \\
-2 k,-2 \ell
\end{array} \mid x, y\right)=\frac{k ! \ell !(a)_{k+\ell}}{(2 k) !(2 \ell) !} x^{k} y^{\ell} F_{3}\left(\begin{array}{c}
k+1, \ell+1 ;-k,-\ell \\
1-a-k-\ell
\end{array} \mid \frac{1}{x}, \frac{1}{y}\right) .
$$

Remark 2.3. The hypergeometric series ${ }_{2} \mathrm{~F}_{1}\left(\begin{array}{c}-k, a \\ -2 k\end{array} \mid x\right)$ is not conventionally defined for a non-negative integer $k$, because of the zero or negative lower parameter. But it can be usefully interpreted in two ways: as a terminating sum of $k+1$ hypergeometric terms, or by taking the term-wise limit with $k \in \mathbb{R}$ approaching a non-negative positive integer. With both interpretations, the ${ }_{2} \mathrm{~F}_{1}$ function is a solution of the respective hypergeometric equation.

In this paper, we adopt the terminating sum interpretation for such ${ }_{2} \mathrm{~F}_{1}$ functions and similar bivariate hypergeometric sums. In particular, the $F_{2}$ function in (2.16) is a sum of $(k+1)(\ell+1)$ hypergeometric terms.

\section{Explicit expressions for dihedral functions}

The following theorem presents generalizations of (1.1)-(1.3). The identities are finite elementary expressions for general dihedral hypergeometric functions. The $F_{2}$ and $F_{3}$ series are finite sums of $(k+1)(\ell+1)$ terms. Because these formulas express solutions of any dihedral hypergeometric equation, we refer to them as canonical.

Note that the $F_{3}$ sum in (3.1) terminates for all (positive or negative) integers $k, \ell$, as the set of upper parameters does not change under the substitutions $k \mapsto-k-1$ and $\ell \mapsto-\ell-1$.

THEOREM 3.1. The following formulas hold for non-negative integers $k, \ell$ and general $a \in \mathbb{C}$ :

$$
\begin{aligned}
& { }_{2} \mathrm{~F}_{1}\left(\begin{array}{c|c}
\frac{a}{2}, \frac{a+1}{2}+\ell \\
a+k+\ell+1
\end{array} \mid 1-z\right)=z^{k / 2}\left(\frac{1+\sqrt{z}}{2}\right)^{-a-k-\ell} \\
& \times F_{3}\left(\begin{array}{c|c}
k+1, \ell+1 ;-k,-\ell & \frac{\sqrt{z}-1}{2 \sqrt{z}}, \frac{1-\sqrt{z}}{2}
\end{array}\right), \\
& \frac{\left(\frac{a+1}{2}\right)_{\ell}}{\left(\frac{1}{2}\right)_{\ell}}{ }_{2} \mathrm{~F}_{1}\left(\begin{array}{c}
\frac{a}{2}, \frac{a+1}{2}+\ell \\
\frac{1}{2}-k
\end{array} \mid z\right)=\frac{(1+\sqrt{z})^{-a}}{2} F_{2}\left(\begin{array}{c|c}
a ;-k,-\ell \\
-2 k,-2 \ell
\end{array} \mid \frac{2 \sqrt{z}}{1+\sqrt{z}}, \frac{2}{1+\sqrt{z}}\right) \\
& +\frac{(1-\sqrt{z})^{-a}}{2} F_{2}\left(\begin{array}{c|c}
a ;-k,-\ell \\
-2 k,-2 \ell
\end{array} \mid \frac{2 \sqrt{z}}{\sqrt{z}-1}, \frac{2}{1-\sqrt{z}}\right),
\end{aligned}
$$




$$
\begin{aligned}
& \frac{\left(\frac{a+1}{2}\right)_{k}\left(\frac{a}{2}\right)_{k+\ell+1}}{\left(\frac{1}{2}\right)_{k}\left(\frac{1}{2}\right)_{k+1}\left(\frac{1}{2}\right)_{\ell}}(-1)^{k} z^{k+\frac{1}{2}}{ }_{2} \mathrm{~F}_{1}\left(\begin{array}{r|r}
\frac{a+1}{2}+k, \frac{a}{2}+k+\ell+1 & z \\
\frac{3}{2}+k &
\end{array}\right) \\
&=\frac{(1-\sqrt{z})^{-a}}{2} F_{2}\left(\begin{array}{c}
a ;-k,-\ell \\
-2 k,-2 \ell
\end{array}\right.\left.\frac{2 \sqrt{z}}{\sqrt{z}-1}, \frac{2}{1-\sqrt{z}}\right) \\
&-\frac{(1+\sqrt{z})^{-a}}{2} F_{2}\left(\begin{array}{c}
a ;-k,-\ell \\
-2 k,-2 \ell
\end{array}\right.
\end{aligned}
$$

Proof. The first identity follows directly from Karlsson's identity [6, Formula 9.4.(90)]; the series on both sides coincide in a neighborhood of $z=1$. The other local solution at $z=1$ is

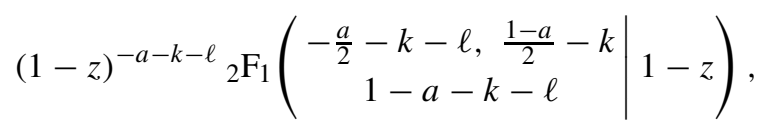

which evaluates to

$$
z^{k / 2}(2-2 \sqrt{z})^{-a-k-\ell} F_{3}\left(\begin{array}{c|c}
k+1, \ell+1 ;-k,-\ell & \frac{\sqrt{z}-1}{2 \sqrt{z}}, \frac{1-\sqrt{z}}{2}
\end{array}\right)
$$

by (3.1) with the substitution $a \mapsto-a-2 k-2 \ell$.

The three terms in (3.2) are solutions of the same second-order Fuchsian equation by Corollary 2.2, so there must be a linear relation between them. Up to a scalar multiple, the right-hand side of (3.2) is the only linear combination of the two $F_{2}$ terms which is invariant under the conjugation $\sqrt{z} \mapsto-\sqrt{z}$. Hence the left- and right-hand sides of (3.2) differ by a factor independent of $z$. Evaluation of the right-hand side at $z=0$ leads to a terminating ${ }_{2} \mathrm{~F}_{1}(2)$ sum. It remains to prove that

$$
{ }_{2} \mathrm{~F}_{1}\left(\begin{array}{c|c}
a,-\ell & 2 \\
-2 \ell & 2
\end{array}\right)=\frac{\left(\frac{a+1}{2}\right)_{\ell}}{\left(\frac{1}{2}\right)_{\ell}} .
$$

Zeilberger's algorithm is by now a routine technique to find two-term hypergeometric identities or recurrence relations for hypergeometric sums (see [5] and [2, Section 3.11]). Its whole output includes certificate information that allows us to check the identity or recurrence relation without computer assistance. Particularly, Zeilberger's algorithm returns the following difference equation for the ${ }_{2} \mathrm{~F}_{1}(2)$ summand $S(\ell, j)$ :

$$
(2 \ell+1) S(\ell+1, j)-(a+1+2 \ell) S(\ell, j)=H(\ell, j+1)-H(\ell, j),
$$

where

$$
S(\ell, j)=\frac{(a)_{j}(-\ell)_{j}}{j !(-2 \ell)_{j}} 2^{j}, \quad H(\ell, j)=-\frac{j(2 \ell+1-j)}{2(\ell+1-j)} S(\ell, j) .
$$

This certificate identity can be checked by hand straightforwardly. To avoid the $0 / 0$ indeterminacy for $j=\ell+1$, we may also write $H(\ell, j)=(1-a-j) S(\ell, j-1)$. While summing up both sides of (3.7) over $j=0,1, \ldots, \ell+1$ we obtain a telescoping sum on the right-hand side that simplifies to $H(\ell, \ell+2)-H(\ell, 0)=0$. The summation of the left-hand side gives a first-order difference equation for the ${ }_{2} \mathrm{~F}_{1}(2)$ sum. The same difference equation is satisfied by the right-hand side of (3.6), and a check that the initial values at $\ell=0$ coincide completes the proof of (3.6). 
As an intermediate step between formulas (3.1), (3.2) and (3.3), we derive formula (3.8) in Lemma 3.2 below. Then we use connection formula [2, (2.3.13)], with both right-hand side terms transformed by Euler's transformation (2.2):

$$
\begin{aligned}
& z^{k+\frac{1}{2}}{ }_{2} \mathrm{~F}_{1}\left(\begin{array}{c|c}
\frac{a+1}{2}+k, \frac{a}{2}+k+\ell+1 \mid z \\
\frac{3}{2}+k
\end{array}\right) \\
& =\frac{\Gamma\left(\frac{3}{2}+k\right) \Gamma(-a-k-\ell)}{\Gamma\left(1-\frac{a}{2}\right) \Gamma\left(\frac{1-a}{2}-\ell\right)}{ }_{2} \mathrm{~F}_{1}\left(\begin{array}{c}
\frac{a}{2}, \frac{a+1}{2}+\ell \\
a+k+\ell+1
\end{array} \mid 1-z\right) \\
& \quad+\frac{\Gamma\left(\frac{3}{2}+k\right) \Gamma(a+k+\ell)}{\Gamma\left(\frac{a+1}{2}+k\right) \Gamma\left(\frac{a}{2}+k+\ell+1\right)}(1-z)^{-a-k-\ell}{ }_{2} \mathrm{~F}_{1}\left(\begin{array}{c}
-\frac{a}{2}-k-\ell, \frac{1-a}{2}-k \\
1-a-k-\ell
\end{array} \mid 1-z\right) .
\end{aligned}
$$

After applying evaluation (3.1) to both right-hand side terms and using relations (2.17) and (3.8), we obtain (3.3).

LEMMA 3.2. We have the following transformation formulas for the terminating $F_{2}$ and $F_{3}$ sums appearing in (3.1)-(3.3):

$$
\begin{aligned}
& (1+\sqrt{z})^{k+\ell} F_{2}\left(\begin{array}{c|c}
a ;-k,-\ell \\
-2 k,-2 \ell
\end{array} \mid \frac{2 \sqrt{z}}{1+\sqrt{z}}, \frac{2}{1+\sqrt{z}}\right) \\
& =\frac{(-1)^{\ell}\left(\frac{a+1}{2}\right)_{\ell}}{\left(\frac{a+1}{2}+k\right)_{\ell}}(1-\sqrt{z})^{k+\ell} F_{2}\left(\begin{array}{c}
-a-2 k-2 \ell ;-k,-\ell \\
-2 k,-2 \ell
\end{array}\right. \\
& F_{3}\left(\begin{array}{c}
k+1, \ell+1 ;-k,-\ell \\
a+k+\ell+1
\end{array} \mid \frac{\sqrt{z}-1}{2 \sqrt{z}}, \frac{1-\sqrt{z}}{2}, \frac{2}{1-\sqrt{z}}\right), \\
& =\frac{(a)_{k+\ell}\left(\frac{a+1}{2}+k\right)_{\ell}}{(1+a+k+\ell)_{k+\ell}\left(\frac{a+1}{2}\right)_{\ell}} F_{3}\left(\begin{array}{c}
k+1, \ell+1 ;-k,-\ell \\
1-a-k-\ell
\end{array}\right.
\end{aligned}
$$

Proof. Connection formula [2, (2.3.13)] gives

$$
\begin{aligned}
{ }_{2} \mathrm{~F}_{1}\left(\begin{array}{c}
\frac{a}{2}, \frac{a+1}{2}+\ell \mid \\
\frac{1}{2}-k
\end{array}\right) \\
=\frac{\Gamma\left(\frac{1}{2}-k\right) \Gamma(-a-k-\ell)}{\Gamma\left(\frac{1-a}{2}-k\right) \Gamma\left(-\frac{a}{2}-k-\ell\right)}{ }_{2} \mathrm{~F}_{1}\left(\begin{array}{c}
\frac{a}{2}, \frac{a+1}{2}+\ell \\
a+k+\ell+1
\end{array} \mid 1-z\right) \\
\quad+\frac{\Gamma\left(\frac{1}{2}-k\right) \Gamma(a+k+\ell)}{\Gamma\left(\frac{a}{2}\right) \Gamma\left(\frac{a+1}{2}+\ell\right)}(1-z)^{-a-k-\ell}{ }_{2} \mathrm{~F}_{1}\left(\begin{array}{c}
-\frac{a}{2}-k-\ell, \frac{1-a}{2}-k \\
1-a-k-\ell
\end{array} \mid 1-z\right) .
\end{aligned}
$$

We evaluate both terms on the right-hand side using (3.1), apply (2.17) twice and compare the whole formula with (3.2). The functions $(1+\sqrt{z})^{-a}$ and $(1-\sqrt{z})^{-a}$ are algebraically independent in general, and identification of respective terms to them gives (3.8).

Formula (3.9) follows from (3.8) via (2.17).

Summarizing, the ${ }_{2} \mathrm{~F}_{1}$ functions (3.2) and (3.3) form a local basis of solutions at $x=0$; the functions in (3.1) and (3.4) form a local basis of solutions at $x=1$; the functions

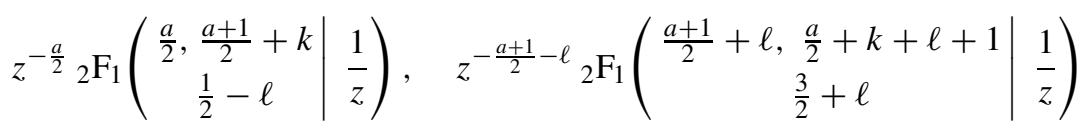


form a local basis of solutions at $x=\infty$. Each of the six functions can be (generally) expressed by a hypergeometric series in four different ways following Euler-Pfaff transformations (2.2) and (2.3). This gives the standard set (and structure) of $6 \times 4=24$ Kummer's hypergeometric solutions of hypergeometric equation (2.1). The symmetries of (2.1) correspond to the permutations of the three singular points and of local exponents at them. Within our context described by (2.10), the action of these permutations on the parameters of hypergeometric functions is the following.

- Permutation of the local exponents at $z=0$ : the parameters are transformed as $k \mapsto-k-1, a \mapsto a+2 k+1$; the hypergeometric solution gets multiplied by $z^{k+1 / 2}$.

- Permutation of the local exponents at $z=1$ : the parameters are transformed as $a \mapsto-a-2 k-2 \ell$; the hypergeometric solution gets multiplied by $(1-z)^{-a-k-\ell}$.

- Permutation of the local exponents at $z=\infty$ : the parameters are transformed as $\ell \mapsto-\ell-1, a \mapsto a+2 \ell+1$; the hypergeometric solution gets multiplied by $z^{-\ell-1 / 2}$.

- Permutation $z \mapsto 1 / z$ of the singularities $z=0, z=\infty$ : the parameters are transformed as $k \leftrightarrow \ell$; the hypergeometric solution gets multiplied by $z^{-a / 2}$.

- Permutation $z \mapsto 1-z$ of the singularities $z=0, z=1$ : the parameters are transformed as $k \mapsto-a-k-\ell-\frac{1}{2}$.

- Permutation $z \mapsto z /(z-1)$ of the singularities $z=1, z=\infty$ : the parameters are transformed as $\ell \mapsto-a-k-\ell-\frac{1}{2}$; the hypergeometric solution gets multiplied by $(1-z)^{-a / 2}$.

Pfaff's fractional-linear transformation (2.3) gives a different relation between the upper parameters in (3.2) or (3.3); for example

$$
{ }_{2} \mathrm{~F}_{1}\left(\begin{array}{c|c}
\frac{a}{2}, \frac{a+1}{2}+\ell \\
\frac{1}{2}-k
\end{array} \mid z\right)=(1-z)^{-\frac{a}{2}}{ }_{2} \mathrm{~F}_{1}\left(\begin{array}{c|c}
\frac{a}{2},-\frac{a}{2}-k-\ell & z \\
\frac{1}{2}-k & \frac{z}{z-1}
\end{array}\right) .
$$

The alternative shape of the upper parameters appears in Section 6.

\section{The simple cases}

In the special cases $k=0$ or $\ell=0$, one of the local exponents of dihedral hypergeometric equation (2.1) is equal to $1 / 2$. Then the terminating double $F_{2}$ or $F_{3}$ sums in (3.1)-(3.3) become terminating single ${ }_{2} \mathrm{~F}_{1}$ sums. For example, special cases of (3.2)and (3.3) are

$$
\begin{aligned}
& { }_{2} \mathrm{~F}_{1}\left(\begin{array}{c}
\frac{a}{2}, \frac{a+1}{2} \\
\frac{1}{2}-k
\end{array} \mid z\right) \\
& \quad=\frac{(1+\sqrt{z})^{-a}}{2}{ }_{2} \mathrm{~F}_{1}\left(\begin{array}{c}
-k, a \\
-2 k
\end{array} \mid \frac{2 \sqrt{z}}{1+\sqrt{z}}\right)+\frac{(1-\sqrt{z})^{-a}}{2}{ }_{2} \mathrm{~F}_{1}\left(\begin{array}{c}
-k, a \\
-2 k
\end{array} \mid \frac{2 \sqrt{z}}{\sqrt{z}-1}\right), \\
& \frac{\left(\frac{a+1}{2}\right) \ell}{\left(\frac{1}{2}\right) \ell}{ }_{2} \mathrm{~F}_{1}\left(\begin{array}{c}
\frac{a}{2}, \frac{a+1}{2}+\ell \mid \\
\frac{1}{2}
\end{array} \mid \begin{array}{l}
z
\end{array}\right) \\
& \quad=\frac{(1+\sqrt{z})^{-a}}{2}{ }_{2} \mathrm{~F}_{1}\left(\begin{array}{c}
-\ell, a \\
-2 \ell
\end{array} \mid \frac{2}{1+\sqrt{z}}\right)+\frac{(1-\sqrt{z})^{-a}}{2}{ }_{2} \mathrm{~F}_{1}\left(\begin{array}{c}
-\ell, a \\
-2 \ell
\end{array} \mid \frac{2}{1-\sqrt{z}}\right),
\end{aligned}
$$




$$
\begin{aligned}
& \frac{(-1)^{k}(a)_{2 k+1}}{2^{2 k+1}\left(\frac{1}{2}\right)_{k}\left(\frac{1}{2}\right)_{k+1}} z^{k+\frac{1}{2}}{ }_{2} \mathrm{~F}_{1}\left(\begin{array}{c|c}
\frac{a+1}{2}+k, \frac{a}{2}+k+1 & z \\
\frac{3}{2}+k & z
\end{array}\right) \\
& =\frac{(1-\sqrt{z})^{-a}}{2}{ }_{2} \mathrm{~F}_{1}\left(\begin{array}{c|c}
-k, a & 2 \sqrt{z} \\
-2 k & \frac{\sqrt{z}-1}{\sqrt{2}}
\end{array}\right)-\frac{(1+\sqrt{z})^{-a}}{2}{ }_{2} \mathrm{~F}_{1}\left(\begin{array}{c|c}
-k, a & 2 \sqrt{z} \\
-2 k & \frac{1+\sqrt{z}}{1+}
\end{array}\right), \\
& \frac{2\left(\frac{a}{2}\right)_{\ell+1}}{\left(\frac{1}{2}\right)_{\ell}} \sqrt{z}{ }_{2} \mathrm{~F}_{1}\left(\begin{array}{c|c}
\frac{a+1}{2}, & \frac{a}{2}+\ell+1 \\
\frac{3}{2} & z
\end{array}\right) \\
& =\frac{(1-\sqrt{z})^{-a}}{2}{ }_{2} \mathrm{~F}_{1}\left(\begin{array}{c|c}
-\ell, a & 2 \\
-2 \ell & \frac{2}{1-\sqrt{z}}
\end{array}\right)-\frac{(1+\sqrt{z})^{-a}}{2}{ }_{2} \mathrm{~F}_{1}\left(\begin{array}{c|c}
-\ell, a \\
-2 \ell
\end{array} \mid \frac{2}{1+\sqrt{z}}\right) .
\end{aligned}
$$

This appearance of terminating ${ }_{2} \mathrm{~F}_{1}$ sums is expectable, since quadratic transformation (2.12) leads to a hypergeometric equation with a cyclic monodromy group. We have quadratic transformations between the dihedral and terminating ${ }_{2} \mathrm{~F}_{1}$ functions. In particular, the identification $z=4 x /(1+x)^{2}$ in the classical formula (2.5) gives

$$
{ }_{2} \mathrm{~F}_{1}\left(\begin{array}{c|c}
\frac{a}{2}, \frac{a+1}{2} & z \\
a+k+1 & z
\end{array}\right)=\left(\frac{1+\sqrt{1-z}}{2}\right)^{-a}{ }_{2} \mathrm{~F}_{1}\left(\begin{array}{c|c}
-k, a & 1-\sqrt{1-z} \\
a+k+1 & 1+\sqrt{1-z}
\end{array}\right),
$$

while formulas (2.4) and (2.3) give

$$
{ }_{2} \mathrm{~F}_{1}\left(\begin{array}{c|c}
\frac{a}{2}, \frac{a+1}{2}+\ell \\
a+\ell+1
\end{array} \mid z\right)=\left(\frac{1+\sqrt{1-z}}{2}\right)^{-a}{ }_{2} \mathrm{~F}_{1}\left(\begin{array}{c|c}
-\ell, a & \sqrt{1-z}-1 \\
a+\ell+1 & \left.\frac{\sqrt{1-z}+1}{\sqrt{1-z}}\right) .
\end{array}\right.
$$

We recognize here special cases of (3.1) after the substitution $z \mapsto 1-z$ and application of Pfaff's fractional-linear transformation (2.3) to the terminating ${ }_{2} \mathrm{~F}_{1}$ series. Relatedly, formulas (3.8) and (3.9) are standard hypergeometric identities when $k=0$ or $\ell=0$.

The dihedral functions with $k=\ell$ can be reduced to the considered case $k \ell=0$ via a quadratic transformation. The quadratic transformation is

$$
E\left(\frac{1}{2}, k+\frac{1}{2}, \lambda\right) \stackrel{2}{\leftarrow} E\left(k+\frac{1}{2}, k+\frac{1}{2}, 2 \lambda\right) .
$$

Quadratic transformations (2.5) and (2.6) give the following expressions:

$$
\begin{aligned}
& { }_{2} \mathrm{~F}_{1}\left(\begin{array}{c|c}
a, a+k+\frac{1}{2} & z \\
\frac{1}{2}-k & z
\end{array}\right)=(1+z)^{-a}{ }_{2} \mathrm{~F}_{1}\left(\begin{array}{c}
\frac{a}{2}, \frac{a+1}{2} \\
\frac{1}{2}-k
\end{array} \mid \frac{4 z}{(1+z)^{2}}\right) \\
& =\frac{(1+\sqrt{z})^{-2 a}}{2}{ }_{2} \mathrm{~F}_{1}\left(\begin{array}{r|r}
-k, a & 4 \sqrt{z} \\
-2 k & \left.\frac{\sqrt{z})^{2}}{(1+\sqrt{2}}\right)
\end{array}\right. \\
& +\frac{(1-\sqrt{z})^{-2 a}}{2}{ }_{2} \mathrm{~F}_{1}\left(\begin{array}{c}
-k, a \\
-2 k
\end{array} \mid-\frac{4 \sqrt{z}}{(\sqrt{z}-1)^{2}}\right), \\
& { }_{2} \mathrm{~F}_{1}\left(\begin{array}{c}
a, a+k+\frac{1}{2} \\
2 a+2 k+1
\end{array} \mid 1-z\right)=\left(\frac{1+z}{2}\right)^{-a}{ }_{2} \mathrm{~F}_{1}\left(\begin{array}{c}
\frac{a}{2}, \frac{a+1}{2} \\
a+k+1
\end{array} \mid \frac{(1-z)^{2}}{(1+z)^{2}}\right) \\
& =z^{k / 2}\left(\frac{1+\sqrt{z}}{2}\right)^{-2 a-2 k}{ }_{2} \mathrm{~F}_{1}\left(\begin{array}{c}
-k, k+1 \\
a+k+1
\end{array} \mid-\frac{(\sqrt{z}-1)^{2}}{4 \sqrt{z}}\right) \text {. }
\end{aligned}
$$


Comparing with formulas (3.2) and (3.1) for the left-hand sides here, we conclude

$$
\begin{aligned}
F_{2}\left(\begin{array}{c}
2 a ;-k,-k \\
-2 k,-2 k
\end{array} \mid x, 2-x\right) & =\frac{\left(a+\frac{1}{2}\right)_{k}}{\left(\frac{1}{2}\right)_{k}}{ }_{2} \mathrm{~F}_{1}\left(\begin{array}{c}
-k, a \\
-2 k
\end{array} \mid x(2-x)\right), \\
F_{3}\left(\begin{array}{c}
-k,-k ; k+1, k+1 \\
2 a+2 k+1
\end{array} \mid x, \frac{x}{2 x-1}\right) & ={ }_{2} \mathrm{~F}_{1}\left(\begin{array}{c}
-k, k+1 \\
a+k+1
\end{array} \mid \frac{x^{2}}{2 x-1}\right) .
\end{aligned}
$$

The finite ${ }_{2} \mathrm{~F}_{1}$ sums can be modified using these transformation formulas [7, Section 7]:

$$
\begin{aligned}
{ }_{2} \mathrm{~F}_{1}\left(\begin{array}{c}
-k, a \\
-2 k
\end{array} \mid x\right) & =(1-x){ }_{2} \mathrm{~F}_{1}\left(\begin{array}{c|c}
-k,-a-2 k \\
-2 k
\end{array} \mid \frac{x}{x-1}\right) \\
& =\frac{k !(a)_{k}}{(2 k) !} x^{k}{ }_{2} \mathrm{~F}_{1}\left(\begin{array}{c}
-k, k+1 \\
1-a-k
\end{array} \mid \frac{1}{x}\right) \\
& =\frac{k !(1+a+k)_{k}}{(2 k) !} x^{k}{ }_{2} \mathrm{~F}_{1}\left(\begin{array}{c}
-k, k+1 \\
1+a+k
\end{array} \mid 1-\frac{1}{x}\right) \\
& =\frac{k !(1+a+k)_{k}}{(2 k) !}{ }_{2} \mathrm{~F}_{1}\left(\begin{array}{c}
-k, a \\
1+a+k
\end{array} \mid 1-x\right) \\
& =\frac{k !(a)_{k}}{(2 k) !}(x-1){ }_{2} \mathrm{~F}_{1}\left(\begin{array}{c}
-k,-a-2 k \\
1-a-k
\end{array} \mid \frac{1}{1-x}\right) .
\end{aligned}
$$

These six hypergeometric expressions have the following arguments after the substitution $x=2 \sqrt{z} /(1+\sqrt{z})$, respectively:

$$
\frac{2 \sqrt{z}}{1+\sqrt{z}}, \quad \frac{2 \sqrt{z}}{\sqrt{z}-1}, \quad \frac{1+\sqrt{z}}{2 \sqrt{z}}, \quad \frac{\sqrt{z}-1}{2 \sqrt{z}}, \quad \frac{\sqrt{z}-1}{\sqrt{z}+1}, \quad \frac{\sqrt{z}+1}{\sqrt{z}-1} .
$$

It is easy to substitute further $z \mapsto 1 / z$ or $z \mapsto 1-z$ here. The substitution $z \mapsto z /(z-1)$ leads to the following six arguments, respectively:

$$
\begin{array}{cc}
2 z-2 \sqrt{z^{2}-z}, \quad 2 z+2 \sqrt{z^{2}-z}, & \frac{1}{2}+\frac{\sqrt{z^{2}-z}}{2 z}, \quad \frac{1}{2}-\frac{\sqrt{z^{2}-z}}{2 z}, \\
1-2 z+2 \sqrt{z^{2}-z}, & 1-2 z-2 \sqrt{z^{2}-z} .
\end{array}
$$

The argument on the right-hand side of (4.6) can be written as

$$
\frac{\sqrt{1-z}-1}{\sqrt{1-z}+1}=1-\frac{2}{z}+\frac{2 \sqrt{1-z}}{z} .
$$

Remark 4.1. As is well known [2, Section 2.9], there are generally 24 hypergeometric series that are solutions of the same hypergeometric equation (2.1); they are referred to as 24 Kummer's solutions. Particularly, a general hypergeometric equation has a basis of hypergeometric solutions at each of the three singular points; the six solutions are different functions, and each of them has four representations as Gauss hypergeometric series due to Euler-Pfaff transformations (2.2) and (2.3). When terminating or logarithmic solutions are present, this structure of 24 solutions degenerates [7].

The hypergeometric equation on the left-hand side of transformation (2.12) has a degenerate structure of 24 Kummer's solutions, as exemplified by formulas (4.12)-(4.16). 
According to [7, Section 7], the same hypergeometric equation has other terminating solution

$$
(1-x)^{-a-k}{ }_{2} \mathrm{~F}_{1}\left(\begin{array}{c}
-k,-a-2 k \\
-2 k
\end{array} \mid x\right)=(1-x)^{-a}{ }_{2} \mathrm{~F}_{1}\left(\begin{array}{c|c}
-k, a & x \\
-2 k & \frac{x}{x-1}
\end{array}\right)
$$

When written in terms of $z$ under the identification $x=2 \sqrt{z} /(1+\sqrt{z})$, both terminating solutions are related (up to a power factor) by the conjugation $\sqrt{z} \mapsto-\sqrt{z}$. The two different terminating solutions are present on the left-hand side of (4.1). Both terminating solutions are representable by six terminating and four non-terminating ${ }_{2} \mathrm{~F}_{1}$ sums. The remaining four Kummer's solutions of the transformed equation are non-terminating ${ }_{2} \mathrm{~F}_{1}$ series at $x=0$. They are related by Euler-Pfaff transformations (2.2)-(2.3), and represent the following solution:

$$
\begin{aligned}
& \frac{(-1)^{k} k !^{2}(a)_{2 k+1}}{(2 k) !(2 k+1) !} x^{2 k+1}{ }_{2} \mathrm{~F}_{1}\left(\begin{array}{c|c}
k+1, a+2 k+1 \\
2 k+2
\end{array} \mid x\right) \\
& =(1-x)^{-a-k}{ }_{2} \mathrm{~F}_{1}\left(\begin{array}{c}
-k,-a-2 k \\
-2 k
\end{array} \mid x\right)-{ }_{2} \mathrm{~F}_{1}\left(\begin{array}{c}
-k, a \\
-2 k
\end{array} \mid x\right) .
\end{aligned}
$$

Quadratic transformation (2.6) gives the following identification:

$$
{ }_{2} \mathrm{~F}_{1}\left(\begin{array}{c|c}
k+1, a+2 k+1 & \frac{2 \sqrt{z}}{1+\sqrt{z}}
\end{array}\right)=(1+\sqrt{z})^{a}{ }_{2} \mathrm{~F}_{1}\left(\begin{array}{c}
\frac{a+1}{2}+k, \frac{a}{2}+k+\ell+1 \\
\frac{3}{2}+k
\end{array} \mid z\right),
$$

consistent with (3.3). Formulas (2.2)-(2.6) for fractional-linear and quadratic transformations may not hold when a degenerate set of 24 Kummer's solutions is involved. In particular, the terminating ${ }_{2} \mathrm{~F}_{1}$ sums in (4.12) and (4.20) are not related by Euler-Pfaff transformations (2.2) and (2.3). As noted in [7, Lemma 3.1], the generic transformations are correct here only if exactly one of the ${ }_{2} \mathrm{~F}_{1}$ sums is interpreted as non-terminating (following Remark 2.3). The right-hand side of (4.21) can be seen as the difference between the terminating and non-terminating interpretations of ${ }_{2} \mathrm{~F}_{1}\left(\begin{array}{c}-k, a \\ -2 k\end{array} \mid x\right)$. Relatedly, there are no two-term identities between the dihedral function in (4.5) and the terminating ${ }_{2} \mathrm{~F}_{1}$ sums in (4.12) or (4.20).

Transformations (2.12) and (4.7) involve degenerate Gauss hypergeometric functions on both sides when $\lambda$ is an integer. We comment further on degeneracies of Kummer's 24 solutions in Subsection 5.4 below.

Remark 4.2. The dihedral functions with $k=0$ or $\ell=0$ can be expressed in terms of the (associated) Legendre functions $P_{\nu}^{\mu}(z), Q_{\nu}^{\mu}(z)$ with integer $v$ or half-integer $\mu$. The relation to the Legendre functions with integer $v$ is clear after comparing the terminating series in (4.13) with the definition $[\mathbf{1}, 8.1 .2]$ :

$$
P_{k}^{\mu}(x)=\frac{1}{\Gamma(1-\mu)}\left(\frac{x+1}{x-1}\right)^{\frac{\mu}{2}}{ }_{2} \mathrm{~F}_{1}\left(\begin{array}{c|c}
-v, v+1 & 1-x \\
1-\mu & \frac{1-x}{2}
\end{array}\right.
$$


By formulas in [1, Chapter 8$]$ or $[\mathbf{4}$, Chapter III] we obtain the following expressions:

$$
\begin{aligned}
& \frac{(2 k-1) ! !}{\Gamma(1-a)} z^{-\frac{k}{2}}(1-z)^{\frac{a+k}{2}}{ }_{2} \mathrm{~F}_{1}\left(\begin{array}{c}
\frac{a}{2}, \frac{a+1}{2} \\
\frac{1}{2}-k
\end{array} \mid z\right) \\
& =\frac{1}{2} P_{k}^{a+k}\left(\frac{1}{\sqrt{z}}\right)+\frac{(-1)^{k}}{2} P_{k}^{a+k}\left(-\frac{1}{\sqrt{z}}\right) \\
& =P_{k}^{a+k}\left(\frac{1}{\sqrt{z}}\right)-\frac{\sin \pi a}{(-1)^{a} \pi} Q_{k}^{a+k}\left(\frac{1}{\sqrt{z}}\right) \text {, } \\
& z^{\frac{k+1}{2}}(1-z)^{\frac{a+k}{2}}{ }_{2} \mathrm{~F}_{1}\left(\begin{array}{c|c}
\frac{a+1}{2}, \frac{a}{2}+k+1 \\
\frac{3}{2}+k
\end{array} \mid z\right) \\
& =\frac{(2 k+1) ! !}{\Gamma(a+2 k+1)}(-1)^{-a-k} Q_{k}^{a+k}\left(\frac{1}{\sqrt{z}}\right), \\
& \frac{2^{\ell+1}\left(\frac{a+1}{2}\right)_{\ell}(1-z)^{\frac{a+\ell}{2}}}{(-1)^{\frac{a-\ell}{2}} \Gamma(1-a)}{ }_{2} \mathrm{~F}_{1}\left(\begin{array}{c|c}
\frac{a}{2}, \frac{a+1}{2}+\ell & z \\
\frac{1}{2} & z
\end{array}\right) \\
& =P_{k}^{a+\ell}(\sqrt{z})+(-1)^{-a-\ell} P_{k}^{a+\ell}(-\sqrt{z}), \\
& \frac{2^{\ell+2}\left(\frac{a}{2}\right)_{\ell+1}(1-z)^{\frac{a+\ell}{2}} \sqrt{z}}{(-1)^{\frac{a-\ell}{2}} \Gamma(1-a)}{ }_{2} \mathrm{~F}_{1}\left(\begin{array}{c|c}
\frac{a+1}{2}, \frac{a}{2}+\ell+1 \\
\frac{3}{2}
\end{array} \mid z\right) \\
& =P_{k}^{a+\ell}(\sqrt{z})-(-1)^{-a-\ell} P_{k}^{a+\ell}(-\sqrt{z}), \\
& z^{-\frac{k}{2}}\left(\frac{1-z}{4}\right)^{\frac{a+k}{2}}{ }_{2} \mathrm{~F}_{1}\left(\begin{array}{c}
\frac{a}{2}, \frac{a+1}{2} \\
a+k+1
\end{array} \mid 1-z\right) \\
& =\Gamma(1+a+k) P_{k}^{-a-k}\left(\frac{1}{\sqrt{z}}\right) .
\end{aligned}
$$

We generally mean $(-1)^{x}=\exp (i \pi x)$ here. For integer $k$, formulas $[1,8.2 .3,8.2 .5]$ give the simpler relation

$$
P_{k}^{-\mu}(x)=(-1)^{k} \frac{\Gamma(1-\mu+k)}{\Gamma(1+\mu+k)} P_{k}^{\mu}(-x),
$$

while $[1,8.2 .4,8.2 .6]$ give

$$
Q_{k}^{\mu}(-x)=(-1)^{k+1} Q_{k}^{\mu}(x), \quad Q_{k}^{-\mu}(x)=(-1)^{-2 \mu} \frac{\Gamma(1-\mu+k)}{\Gamma(1+\mu+k)} Q_{k}^{\mu}(x) .
$$

Expressions in terms of Legendre functions with half-integer $\mu$ are obtained by applying [1, 8.2.7-8]:

$$
\begin{aligned}
& P_{-\mu-\frac{1}{2}}^{-k-\frac{1}{2}}\left(\frac{1}{\sqrt{1-z}}\right)=\frac{(-1)^{-\mu} \sqrt{2 / \pi}}{\Gamma(\mu+k+1)}\left(\frac{1-z}{z}\right)^{\frac{1}{4}} Q_{k}^{\mu}\left(\frac{1}{\sqrt{z}}\right), \\
& Q_{-\mu-\frac{1}{2}}^{-k-\frac{1}{2}}\left(\frac{1}{\sqrt{1-z}}\right)=i(-1)^{k+1} \Gamma(-\mu-k) \sqrt{\frac{\pi}{2}}\left(\frac{1-z}{z}\right)^{\frac{1}{4}} P_{k}^{\mu}\left(\frac{1}{\sqrt{z}}\right) .
\end{aligned}
$$




\section{Degenerate and logarithmic solutions}

So far we have considered solutions of hypergeometric equations $E(k+1 / 2, \ell+1 / 2, \lambda)$, where $k, \ell$ are integers but $\lambda$ is not an integer. If the third local exponent difference $\lambda$ is an integer, the monodromy group is either completely reducible and isomorphic to $\mathbb{Z} / 2 \mathbb{Z}$ or (in the presence of logarithmic solutions) it is isomorphic to an infinite dihedral group. First, we state conditions for how to separate the two cases.

\subsection{Conditions for logarithmic solutions}

THEOREM 5.1. Let $k, \ell, n$ denote non-negative integers. Then equation $E(k+1 / 2$, $\ell+1 / 2, n)$ has logarithmic solutions if and only if

$$
\begin{aligned}
& n \leq k+\ell, \quad \text { if } n+k+\ell \text { is even, } \\
& n<|k-\ell|, \quad \text { if } n+k+\ell \text { is odd. }
\end{aligned}
$$

If this is the case, the monodromy group of (2.1) is an infinite dihedral group; otherwise the monodromy group is isomorphic to $\mathbb{Z} / 2 \mathbb{Z}$.

Proof. A representative equation (2.1) with the assumed local exponents has

$$
A=-\frac{n+k+\ell}{2}, \quad B=-\frac{n+k-\ell-1}{2}, \quad C=\frac{1}{2}-k .
$$

The sequence $A, 1-B, C-A, 1+B-C$ contains exactly two integers. By part (3) of [7, Theorem 2.2], there are no logarithmic solutions precisely when the two integers are either both positive or both non-positive. Equivalently, there are logarithmic solutions precisely when one of the integers is positive while the other is non-positive. If $n+k+\ell$ is even, the two integers are

$$
A=-\frac{n+k+\ell}{2} \text { and } 1+B-C=1+\frac{k+\ell-n}{2} .
$$

The first integer is always zero or negative; the second integer is positive exactly when $n \leq k+\ell$. If $n+k+\ell$ is odd, the two integers are

$$
1-B=\frac{1+n+k-\ell}{2} \text { and } C-A=\frac{1+n-k+\ell}{2} .
$$

We may assume $\ell \leq k$ without loss of generality. Then the first integer is positive; the second integer is non-positive exactly when $n<k-\ell$.

For comparison, recall [7, Section 9] that the hypergeometric equation $E(k, \ell, n)$ with non-negative integers $k, \ell, n$ has logarithmic solutions if and only if one of the integers is greater than the sum of the other two. Here is a more direct formulation of Theorem 5.1.

COROLlary 5.2. Suppose that $p, q$ are half-integers, and $n$ is a non-negative integer. The set $\{|p-q|,|p+q|\}$ contains two integers of different parity; let $K$ be the integer in this set such that $K+n$ is odd. Then equation $E(p, q, n)$ has the monodromy group isomorphic to $\mathbb{Z} / 2 \mathbb{Z}$ if $K<n$, and it has logarithmic solutions otherwise.

Here is a formulation of Theorem 5.1 that refers to the parameter $a=\lambda+k+\ell$ in (2.10) rather than to the local exponent difference $\lambda$. 
COROLlary 5.3. Let $k, \ell, m$ denote non-negative integers, and suppose that $k \leq \ell$. Hypergeometric equation (2.1) with (2.10) and $a=-m$ has logarithmic solutions if and only if

$$
\begin{aligned}
& 0 \leq \frac{m}{2} \leq k+\ell, \quad \text { for even } m, \\
& k<\frac{m+1}{2} \leq \ell, \quad \text { for odd } m .
\end{aligned}
$$

If this is the case, the monodromy group of (2.1) is an infinite dihedral group; otherwise the monodromy group is isomorphic to $\mathbb{Z} / 2 \mathbb{Z}$.

Proof. Theorem 5.1 is being applied to equation $E(k+1 / 2, \ell+1 / 2, m-k-\ell)$ or to $E(k+1 / 2, \ell+1 / 2, k+\ell-m)$.

We refer to solutions of a hypergeometric equation with the monodromy group $\mathbb{Z} / 2 \mathbb{Z}$ as degenerate. Degenerate or logarithmic solutions are involved exactly when a Pochhammer factor on the left-hand side of our main identities (3.2) and (3.3) vanishes. The identities are still valid even if the left-hand side vanishes. Recalling $\lambda=a-k-\ell$, we set

$$
K_{1}=\min (k, \ell), \quad K_{2}=\ell-k, \quad L=k+\ell .
$$

Then Pochhammer factors in both (3.2) and (3.3) vanish if and only if

$$
\begin{aligned}
& a \in\left\{-1,-3, \ldots, 1-2 K_{1}\right\}, \\
& \lambda \in\left\{\left|K_{2}\right|+1,\left|K_{2}\right|+3, \ldots, L-1\right\} .
\end{aligned}
$$

Only a Pochhammer factor in (3.3) vanishes if and only if

$$
\begin{aligned}
& a \in\{0,-2, \ldots,-2 L\} \cup\left\{-1-2 K_{1},-3-2 K_{1}, \ldots, 1-2 k\right\}, \\
& \lambda \in\{-L, 2-L, \ldots, L\} \cup\left\{K_{2}+1, K_{2}+3, \ldots,\left|K_{2}\right|-1\right\},
\end{aligned}
$$

and only the Pochhammer factor in (3.2) vanishes if and only if

$$
\begin{aligned}
& a \in\left\{-1-2 K_{1},-3-2 K_{1}, \ldots, 1-2 \ell\right\}, \\
& \lambda \in\left\{-K_{2}+1,-K_{2}+3, \ldots,\left|K_{2}\right|-1\right\} .
\end{aligned}
$$

By Theorem 5.1, the hypergeometric equation $E(k+1 / 2, \ell+1 / 2, \lambda)$ has logarithmic solutions if and only if exactly one of conditions (5.9) or (5.11) holds. Equivalently, by Corollary 5.3 we have to check whether exactly one of conditions (5.8) or (5.10) holds.

\subsection{The monodromy group $\mathbb{Z} / 2 \mathbb{Z}$}

By Corollary 5.3, the monodromy group of hypergeometric equation (2.1) with (2.10) and $a=-m$ is isomorphic to $\mathbb{Z} / 2 \mathbb{Z}$ if and only if either (5.6) holds or both (5.8) and (5.10) hold. In the former case, formulas (3.2) and (3.3) do not give terminating expressions for the functions

$$
{ }_{2} \mathrm{~F}_{1}\left(\begin{array}{c|c}
-\frac{m}{2}, \ell-\frac{m-1}{2} & z \\
\frac{1}{2}-k & z
\end{array}\right), \quad{ }_{2} \mathrm{~F}_{1}\left(\begin{array}{c|c}
k-\frac{m-1}{2}, & k+\ell-\frac{m}{2}+1 \\
\frac{3}{2}+k & z
\end{array}\right) .
$$


But terminating expressions for these functions are obtained by applying Euler's transformation (2.2). Adding up the right-hand sides of (3.2) and (3.3) suggests the following lemma.

LEMMA 5.4. Suppose that $m$ is an odd positive integer. Then for any $b, c \in \mathbb{C}$ the terminating sum

$$
F_{2}\left(\begin{array}{c|c}
-m ; b, c & x, 2-x \\
2 b, 2 c &
\end{array}\right)
$$

is identically zero.

Proof. Here we have a triangular $p \geq 0, q \geq 0, p+q \leq m$ terminating sum (2.13), as opposed to rectangular $0 \leq p \leq k, 0 \leq q \leq \ell$ terminating sums frequent in this article.

The $F_{2}(x, 2-x)$ sum is a rational function in $b, c$ of degree at most $2 m$. It is enough to show that this rational function is zero for infinitely many values of $b, c$. By (3.2) and (3.3), the $F_{2}$ sum is zero when $b, c$ are integers $\leq-(m+1) / 2$.

We have the monodromy group $\mathbb{Z} / 2 \mathbb{Z}$ also in the cases $a=-m$ with odd $m>$ $2 \max (k, \ell)$ or even $m>2(k+\ell)$. Then the hypergeometric functions in (5.12) themselves terminate, while a non-terminating ${ }_{2} \mathrm{~F}_{1}$ solution is (3.4). Theorem 5.4 does not apply to the $F_{2}$ functions in (3.2) and (3.3) for odd $m>2 \min (k, \ell)$ because, generally,

$$
\lim _{b \rightarrow-k} \frac{(-m)_{i+j}(b)_{i}(c)_{j}}{i ! j !(2 b)_{i}(2 c)_{j}} \neq 0 \quad \text { for } 2 k<i \leq m
$$

in the triangular sum of Theorem 5.4, while the same term is taken to be zero in the rectangular sums in (3.2) and (3.3).

\subsection{Logarithmic solutions}

By Corollary 5.3, hypergeometric equation (2.1) with (2.10) and $a=-m$ has logarithmic solutions if and only if exactly one of the conditions (5.8) or (5.10) holds. Then one of the equations (3.2) or (3.3) vanishes, and the other is a terminating hypergeometric sum. The terminating sum is a non-logarithmic solution, obviously. We have

$$
\frac{\left(\frac{1-m}{2}\right)_{\ell}}{\left(\frac{1}{2}\right)_{\ell}}{ }_{2} \mathrm{~F}_{1}\left(\begin{array}{c|c}
-\frac{m}{2}, \ell-\frac{m-1}{2} & z \\
\frac{1}{2}-k & z
\end{array}\right)=(1-\sqrt{z})^{m} F_{2}\left(\begin{array}{c}
-m ;-k,-\ell \\
-2 k,-2 \ell
\end{array} \mid \frac{2 \sqrt{z}}{\sqrt{z}-1}, \frac{2}{\sqrt{z}-1}\right)
$$

in the case (5.8) with $a=-m$, since both summands on the right-hand side of (3.2) are equal. We have the same right-hand side expression for the left-hand side of (3.3) in the case (5.10) with $a=-m$. The ${ }_{2} \mathrm{~F}_{1}$ function in the vanishing equation (3.2) or (3.3) has a logarithmic expression that can be obtained by differentiating both sides of the vanishing equation with respect to $a$ and evaluating at $a=-m$. We have

$$
\frac{d}{d a}(1+\sqrt{z})^{-a}=-(1+\sqrt{z})^{-a} \log (1+\sqrt{z})
$$

and similarly for $(d / d a)(1-\sqrt{z})^{-a}$. On the left-hand side of the vanishing equation (3.2) or (3.3) we have exactly one (Pochhammer) factor vanishing; to differentiate the product, it is enough to differentiate only the vanishing Pochhammer symbol. 
LEMma 5.5. The formula for differentiating a Pochhammer symbol is

$$
\frac{d}{d a}(a)_{N}= \begin{cases}(a)_{N}\left(\frac{1}{a}+\frac{1}{a+1}+\cdots+\frac{1}{a+N-1}\right), & \text { if }(a)_{N} \neq 0, \\ (-1)^{a}(-a) !(N+a-1) !, & \text { if }(a)_{N}=0 .\end{cases}
$$

Proof. If $(a)_{N} \neq 0$, we are differentiating a product of $N$ linear functions in $a$. If $(a)_{N}=0$, then $a$ is zero or a negative integer. Writing $a=-m+\epsilon$ we have

$$
(a)_{N}=(-m+\epsilon)(1-m+\epsilon) \cdots(-1+\epsilon) \epsilon(1+\epsilon) \cdots(N-m-1+\epsilon) .
$$

The differentiation is equivalent to dividing out $\epsilon$ and setting $\epsilon=0$.

Let $\psi(x)$ denote the digamma function, $\psi(x)=\Gamma^{\prime}(x) / \Gamma(x)$. The sum

$$
\frac{1}{a}+\frac{1}{a+1}+\cdots+\frac{1}{a+N-1}
$$

can be written as $\psi(a+N)-\psi(a)$ if $a$ is not zero or a negative integer. For an integer $m \geq 0$ let us define the derivative Pochhammer symbol

$$
(-m)_{N}^{\dagger}=\left.\frac{d}{d a}(a)_{N}\right|_{a=-m}
$$

By Lemma 5.5 we have

$$
(-m)_{N}^{\dagger}= \begin{cases}(-m)_{N}(\psi(m+1-N)-\psi(m+1)), & \text { if } N \leq m, \\ (-1)^{m} m !(N-m-1) !, & \text { if } N>m .\end{cases}
$$

Note that $(-m)_{N}^{\dagger} \neq 0$ when $N>0$. On the other hand, $(-m)_{0}^{\dagger}=0$ even if $m=0$.

When differentiating both sides of (3.2) or (3.3) with respect to $a$, we keep in mind that $(d / d a)(a / 2)_{N}$ evaluates to $\frac{1}{2}(-m / 2)_{N}^{\dagger}$. For even $a=-m$ satisfying (5.8), we differentiate (3.3), use (5.14) and obtain

$$
\begin{aligned}
& \frac{\left(\frac{1-m}{2}\right)_{k}\left(\frac{m}{2}\right) !\left(k+\ell-\frac{m}{2}\right) !}{\left(\frac{1}{2}\right)_{k}\left(\frac{1}{2}\right)_{k+1}\left(\frac{1}{2}\right)_{\ell}}(-1)^{k+\frac{m}{2}} z^{k+\frac{1}{2}}{ }_{2} \mathrm{~F}_{1}\left(\begin{array}{r}
\left.k-\frac{m-1}{2}, k+\ell-\frac{m}{2}+1 \mid z\right) \\
\frac{3}{2}+k
\end{array} \mid z\right) \\
& =\frac{\left(\frac{1-m}{2}\right)_{\ell}}{\left(\frac{1}{2}\right)_{\ell}} \log \frac{1+\sqrt{z}}{1-\sqrt{z}}{ }_{2} \mathrm{~F}_{1}\left(\begin{array}{c}
-\frac{m}{2}, \ell-\frac{m-1}{2} \\
\frac{1}{2}-k
\end{array} \mid z\right) \\
& +(1-\sqrt{z})^{m} F_{2}^{\dagger}\left(\begin{array}{c}
-m ;-k,-\ell \\
-2 k,-2 \ell
\end{array} \mid \frac{2 \sqrt{z}}{\sqrt{z}-1}, \frac{2}{1-\sqrt{z}}\right) \\
& -(1+\sqrt{z})^{m} F_{2}^{\dagger}\left(\begin{array}{c}
-m ;-k,-\ell \\
-2 k,-2 \ell
\end{array} \mid \frac{2 \sqrt{z}}{1+\sqrt{z}}, \frac{2}{1+\sqrt{z}}\right) .
\end{aligned}
$$

Here the $F_{2}^{\dagger}$ functions are rectangular sums of $(k+1)(\ell+1)$ terms, defined as the $F_{2}$ sums in (2.13) but with each Pochhammer symbol $(-m)_{i+j}$ replaced by the derivative $(-m)_{i+j}^{\dagger}$ following (5.18). For odd $a=-m$ satisfying (5.8), the differentiation of (3.3) gives the same right-hand side, but the left-hand side is

$$
\frac{\left(\frac{m-1}{2}\right) !\left(k-\frac{m+1}{2}\right) !\left(-\frac{m}{2}\right)_{k+\ell+1}}{\left(\frac{1}{2}\right)_{k}\left(\frac{1}{2}\right)_{k+1}\left(\frac{1}{2}\right)_{\ell}}(-1)^{k+\frac{m-1}{2}} z^{k+\frac{1}{2}}{ }_{2} \mathrm{~F}_{1}\left(\begin{array}{c}
k-\frac{m-1}{2}, k+\ell-\frac{m}{2}+1 \\
\frac{3}{2}+k
\end{array} \mid z\right) .
$$


For odd $a=-m$ satisfying (5.10), differentiation of (3.2) gives

$$
\begin{aligned}
& \frac{\left(\frac{m-1}{2}\right) !\left(\ell-\frac{m+1}{2}\right) !}{\left(\frac{1}{2}\right)_{\ell}}(-1)^{\frac{m-1}{2}}{ }_{2} \mathrm{~F}_{1}\left(\begin{array}{c}
-\frac{m}{2}, \ell-\frac{m-1}{2} \\
\frac{1}{2}-k
\end{array} \mid z\right) \\
& =\frac{\left(\frac{1-m}{2}\right)_{k}\left(-\frac{m}{2}\right)_{k+\ell+1}}{\left(\frac{1}{2}\right)_{k}\left(\frac{1}{2}\right)_{k+1}\left(\frac{1}{2}\right)_{\ell}}(-1)^{k} z^{k+\frac{1}{2}} \log \frac{1+\sqrt{z}}{1-\sqrt{z}}{ }_{2} \mathrm{~F}_{1}\left(\begin{array}{c}
k-\frac{m-1}{2}, k+\ell-\frac{m}{2}+1 \\
\frac{3}{2}+k
\end{array} \mid z\right) \\
& +(1+\sqrt{z})^{m} F_{2}^{\dagger}\left(\begin{array}{c|c}
-m ;-k,-\ell & \frac{2 \sqrt{z}}{1+\sqrt{z}}, \frac{2}{1+\sqrt{z}}
\end{array}\right) \\
& +(1-\sqrt{z})^{m} F_{2}^{\dagger}\left(\begin{array}{c|c}
-m ;-k,-\ell & 2 \sqrt{z} \\
-2 k,-2 \ell & \frac{2}{\sqrt{z}-1}, \frac{2}{1-\sqrt{z}}
\end{array}\right) \text {. }
\end{aligned}
$$

As we will see in Section 6, nice expressions for our dihedral ${ }_{2} \mathrm{~F}_{1}$ functions are obtained under the trigonometric substitution $z \mapsto-\tan ^{2} x$. In particular, one may recognize

$$
\arctan x=\frac{1}{2 i} \log \frac{1+i x}{1-i x} .
$$

\subsection{Degeneration of 24 Kummer's solutions}

As observed in Remark 4.1, the structure of 24 Kummer's solutions degenerates if the hypergeometric equation has terminating or logarithmic solutions. Particularly, not all 24 Kummer's solutions are well defined or distinct when logarithmic solutions are present.

In the case of the monodromy group $\mathbb{Z} / 2 \mathbb{Z}$, the degenerate structure is described in [7, Section 7]. The two solutions in (5.12) can be represented by terminating ${ }_{2} \mathrm{~F}_{1}$ sums in six ways (with any of the arguments $z, z /(z-1), 1 / z, 1 /(1-z), 1-1 / z, 1-z)$ and by nonterminating ${ }_{2} \mathrm{~F}_{1}$ series (around $z=0$ or $z=\infty$ ) in four ways. The remaining four of the 24 Kummer's solutions represent, up to a power factor, the function in (3.1). Terminating $F_{3}$ expression (3.1) holds, but that function can be expressed as a linear combination of two terminating ${ }_{2} \mathrm{~F}_{1}$ solutions. For example, the following $z \mapsto 1-z$ version of [7, (43)] can be used:

$$
\begin{aligned}
& (1-z)^{n+m+1}{ }_{2} \mathrm{~F}_{1}\left(\begin{array}{c|c}
a+n+1, m+1 \\
n+m+2
\end{array} \mid 1-z\right) \\
& =\frac{(n+1)_{m+1}}{(-a)_{m+1}}{ }_{2} \mathrm{~F}_{1}\left(\begin{array}{c|c}
-n, a-m \\
1+a
\end{array} \mid z\right)+\frac{(m+1)_{n+1}}{(a)_{n+1}} z^{-a}{ }_{2} \mathrm{~F}_{1}\left(\begin{array}{c}
-m,-a-n \\
1-a
\end{array} \mid z\right) \text {, }
\end{aligned}
$$

with

$$
\begin{array}{ll}
(n, m, a) \mapsto\left(k-\frac{m+1}{2}, \ell-\frac{m+1}{2},-k-\frac{1}{2}\right), & \text { for odd } m<2 \min (k, \ell), \\
(n, m, a) \mapsto\left(\frac{m-1}{2}-\ell, \frac{m-1}{2}-k,-k-\frac{1}{2}\right), & \text { for odd } m>2 \max (k, \ell), \\
(n, m, a) \mapsto\left(\frac{m}{2}, \frac{m}{2}-k-\ell-1,-k-\frac{1}{2}\right), & \text { for even } m>2(k+\ell) .
\end{array}
$$


The logarithmic case with terminating solutions is described in [7, Section 6]. There are 20 distinct hypergeometric series, or less if $m=k+\ell$. Among them, there are eight terminating and four non-terminating hypergeometric series representing the non-logarithmic terminating solution, as in (5.14). The remaining eight (in general) Kummer's solutions are non-terminating series around $z=0$ or $z=\infty$ and represent two different functions, in particular the logarithmic solution among (5.19)-(5.21). The logarithmic solution can be expressed following [7, Theorem 6.1] as well. Formulas [7, (36) and (38)] give the generic identity

$$
\begin{aligned}
& \frac{(-1)^{m+1} z^{n+m+1-a}}{(1-a)_{n+m+1}}{ }_{2} \mathrm{~F}_{1}\left(\begin{array}{c}
m+1-a, n+m+1 \\
n+m+2-a
\end{array} \mid z\right) \\
& =\frac{(m+1-a)_{n}}{n !(n+m) !}{ }_{2} \mathrm{~F}_{1}\left(\begin{array}{c}
-n, a \\
a-n-m
\end{array} \mid z\right)(\log (1-z)+\pi \cot \pi a) \\
& \quad-\frac{(z-1)^{-m}}{(1-a)_{m}} \sum_{j=0}^{m-1} \frac{(a-m)_{j}(m-j-1) !}{(n+m-j) ! j !}(1-z)^{j} \\
& \quad+\sum_{j=0}^{n} \frac{(a)_{j}(\psi(a+j)+\psi(n-j+1)-\psi(m+j+1)-\psi(j+1))}{(m+j) !(n-j) ! j !}(z-1)^{j} \\
& \quad+(-1)^{n} \sum_{j=n+1}^{\infty} \frac{(a)_{j}(j-n-1) !}{(m+j) ! j !}(1-z)^{j}
\end{aligned}
$$

where we should substitute

$$
\begin{aligned}
&(n, m, a) \mapsto\left(\frac{m}{2}, k+\ell-m, \ell-\frac{m-1}{2}\right), \quad \text { if } m \text { is even, } 0 \leq m \leq k+\ell, \\
&(n, m, a) \mapsto\left(k+\ell-\frac{m}{2}, m-k-\ell, \frac{m+1}{2}-k\right), \quad \text { if } m \text { is even, } k+\ell \leq m \leq 2(k+\ell), \\
&(n, m, a) \mapsto\left(\frac{m-1}{2}-\ell, k+\ell-m,-\frac{m}{2}\right), \quad \text { if } m \text { is odd, } 2 \min (k, \ell)<m<2 k, \\
&(n, m, a) \mapsto\left(\frac{m-1}{2}-k, k+\ell-m, k+\ell-\frac{m}{2}+1\right), \\
& \quad \text { if } m \text { is odd, } 2 \min (k, \ell)<m<2 \ell,
\end{aligned}
$$

to get the same ${ }_{2} \mathrm{~F}_{1}$ function on the left-hand side as in (5.19)-(5.21). The cot $\pi a$ term is zero when $a$ is a half-integer in (5.24). These formulas do not contain double sums or square roots, but the last term in (5.24) is a non-terminating series.

Remark 5.6. As observed in Section 4, the terminating $F_{2}$ or $F_{3}$ sums in (3.1)-(3.3) become terminating ${ }_{2} \mathrm{~F}_{1}$ sums in the case $k=0$ or $\ell=0$. If, additionally, $a$ is an integer, the structure of 24 Kummer's solutions for the quadratically transformed equation degenerates further. If $a=-m$ with $0 \leq m \leq 2 k$, there are logarithmic solutions by Corollary 5.3. We then have to apply [7, Section 9] with $(n, m, \ell)=(|k-m|,|k-m|, m)$ to the transformed ${ }_{2} \mathrm{~F}_{1}$ sums. There are at most ten different terminating ${ }_{2} \mathrm{~F}_{1}$ solutions, all representing the same elementary solution of the transformed equation. 
If $a$ is an integer and $|a+k|>k$, the dihedral group degenerates to $\mathbb{Z} / 2 \mathbb{Z}$, while the monodromy group of the quadratically transformed equation is trivial. By [7, Section 8], the transformed equation has three solutions like as in (4.12), each representable by six terminating and two non-terminating ${ }_{2} \mathrm{~F}_{1}$ series.

\section{Trigonometric expressions}

Formulas (1.6) and (1.7) show attractive trigonometric expressions for the simplest dihedral functions. Analogous trigonometric expressions are possible for all dihedral functions, if only due to contiguous relations. The literature appears to give only trigonometric expressions for the simplest dihedral functions. In particular (see $[1,15.1]$ and $[4,2.8])$, here are fractionallinear transformations of (1.6) and (1.7):

$$
\begin{aligned}
{ }_{2} \mathrm{~F}_{1}\left(\begin{array}{c}
\frac{1+a}{2}, \frac{1-a}{2} \mid \\
\frac{1}{2}
\end{array} \sin ^{2} x\right) & =\frac{\cos a x}{\cos x}, \\
{ }_{2} \mathrm{~F}_{1}\left(\begin{array}{c}
\frac{2+a}{2}, \frac{2-a}{2} \mid \\
\frac{3}{2}
\end{array} \mid \sin ^{2} x\right) & =\frac{2 \sin a x}{a \sin 2 x}, \\
{ }_{2} \mathrm{~F}_{1}\left(\frac{a}{2}, \frac{a+1}{2} \mid-\tan ^{2} x\right) & =\cos a x \cos ^{a} x, \\
\frac{1}{2} \mid & { }_{2} \mathrm{~F}_{1}\left(\frac{a+1}{2}, \frac{a+2}{2} \mid-\tan ^{2} x\right)=\frac{\sin a x}{a \sin x} \cos ^{a+1} x .
\end{aligned}
$$

According to the latter two formulas, we should use the argument substitution $z \mapsto-\tan ^{2} x$ in our main expressions. Nicer expressions are obtained after fractional-linear transformation (2.3), hence with the argument $\sin ^{2} x$.

\subsection{General trigonometric expressions}

The following theorem gives trigonometric versions of formulas (3.2) and (3.3) after Pfaff's transformation (2.3). Subsequently, we discuss here simplification of trigonometric formulas. Trigonometric modification of formula (3.1) is discussed in Subsection 6.4.

THEOREM 6.1. Let us denote

$$
\Upsilon_{p, q}^{a, k, \ell}(x):=\frac{2^{p+q}(-k)_{p}(-\ell)_{q}(a)_{p+q}}{(-2 k)_{p}(-2 \ell)_{q} p ! q !} \sin ^{p} x \cos ^{q} x .
$$

Then

$$
\begin{aligned}
& \frac{\left(\frac{a+1}{2}\right)_{\ell}}{\left(\frac{1}{2}\right)_{\ell}}{ }_{2} \mathrm{~F}_{1}\left(\begin{array}{c|c}
\frac{a}{2}, & -\frac{a}{2}-k-\ell \\
\frac{1}{2}-k & \sin ^{2} x
\end{array}\right) \\
& =\sum_{p=0}^{k} \sum_{q=0}^{\ell} \Upsilon_{p, q}^{a, k, \ell}(x) \cos \left(a x+(p+q) x-\frac{\pi}{2} p\right),
\end{aligned}
$$




$$
\begin{array}{r}
\frac{\left(\frac{a+1}{2}\right)_{k}\left(\frac{a}{2}\right)_{k+\ell+1} \sin ^{2 k+1} x}{\left(\frac{1}{2}\right)_{k}\left(\frac{1}{2}\right)_{k+1}\left(\frac{1}{2}\right)_{\ell}}{ }_{2} \mathrm{~F}_{1}\left(\begin{array}{c}
\frac{a+1}{2}+k, \frac{1-a}{2}-\ell \\
\frac{3}{2}+k
\end{array} \mid \sin ^{2} x\right) \\
=\sum_{p=0}^{k} \sum_{q=0}^{\ell} \Upsilon_{p, q}^{a, k, \ell}(x) \sin \left(a x+(p+q) x-\frac{\pi}{2} p\right) .
\end{array}
$$

Proof. After fractional-linear transformation (2.3) and the substitution $z \mapsto-\tan ^{2} x$ in (3.2) we obtain

$$
\begin{aligned}
& \frac{\left(\frac{a+1}{2}\right)_{\ell}}{\left(\frac{1}{2}\right)_{\ell}}{ }_{2} \mathrm{~F}_{1}\left(\begin{array}{c|c}
\frac{a}{2},-\frac{a}{2}-k-\ell \\
\frac{1}{2}-k
\end{array} \mid \sin ^{2} x\right) \\
& =\frac{\exp (-i a x)}{2} F_{2}\left(\begin{array}{c|c}
a ;-k,-\ell & 2 i \sin x \\
-2 k,-2 \ell & \frac{2 \cos x}{\exp i x}, \frac{\exp i x}{\operatorname{ex}}
\end{array}\right)
\end{aligned}
$$

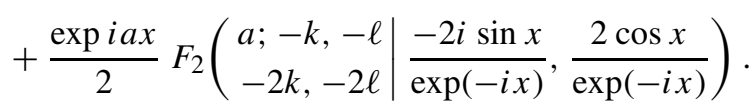

We use Euler's formula exp $i x=\cos x+i \sin x$ as well. We add the two double sums term by term, and after the identification $\pm 2 i=2 \exp ( \pm i \pi / 2)$ we obtain the double sum in (6.6). Note that $\Upsilon_{p, q}^{a, k, \ell}(x)$ is the generic summand of

$$
F_{2}\left(\begin{array}{c}
a ;-k,-\ell \\
-2 k,-2 \ell
\end{array} \mid 2 \sin x, 2 \cos x\right) .
$$

Formula (6.7) follows similarly. Withal, the substitution $z \mapsto-\tan ^{2} x$ into $(-1)^{k} z^{k+1 / 2}$ gives $i \sin ^{2 k+1} x / \cos ^{2 k+1} x$.

The right-hand side of (6.6) can be written as

$$
P_{k, \ell}(x) \cos a x+Q_{k, \ell}(x) \sin a x,
$$

with

$$
\begin{aligned}
P_{k, \ell}(x) & =\sum_{p=0}^{k} \sum_{q=0}^{\ell} \Upsilon_{p, q}^{a, k, \ell}(x) \cos \left(\frac{\pi}{2} p-(p+q) x\right), \\
Q_{k, \ell}(x) & =\sum_{p=0}^{k} \sum_{q=0}^{\ell} \Upsilon_{p, q}^{a, k, \ell}(x) \sin \left(\frac{\pi}{2} p-(p+q) x\right) .
\end{aligned}
$$

The right-hand side of (6.7) can then be written as

$$
P_{k, \ell}(x) \sin a x-Q_{k, \ell}(x) \cos a x,
$$

and we have

$$
P_{\ell, k}(x)=P_{k, \ell}\left(\frac{\pi}{2}-x\right), \quad Q_{\ell, k}(x)=-Q_{k, \ell}\left(\frac{\pi}{2}-x\right) .
$$

Generally, here are the first few terms of the double sums $P_{k, \ell}(x), Q_{k, \ell}(x)$ :

$$
\begin{aligned}
P_{k, \ell}(x)=1+ & a \cos ^{2} x+\frac{a(a+1)(\ell-1)}{2 \ell-1} \cos ^{2} x \cos 2 x \\
+ & \frac{a(a+1)(a+2)(\ell-2)}{3(2 \ell-1)} \cos ^{3} x \cos 3 x+\cdots
\end{aligned}
$$




$$
\begin{aligned}
& +a \sin ^{2} x+\frac{a(a+1)}{2} \sin ^{2} 2 x \\
& +\frac{a(a+1)(a+2)(\ell-1)}{2 \ell-1} \sin x \cos ^{2} x \sin 3 x+\cdots \\
& -\frac{a(a+1)(k-1)}{2 k-1} \sin ^{2} x \cos 2 x \\
& -\frac{a(a+1)(a+2)(k-1)}{2 k-1} \sin ^{2} x \cos x \cos 3 x+\cdots \\
& -\frac{a(a+1)(a+2)(k-2)}{3(2 k-1)} \sin ^{3} x \sin 3 x-\cdots \\
& +\cdots, \\
& Q_{k, \ell}(x)=0-\frac{a}{2} \sin 2 x-\frac{a(a+1)(\ell-1)}{2 \ell-1} \cos ^{2} x \sin 2 x \\
& -\frac{a(a+1)(a+2)(\ell-2)}{3(2 \ell-1)} \cos ^{3} x \sin 3 x-\cdots \\
& +\frac{a}{2} \sin 2 x+\frac{a(a+1)}{4} \sin 4 x \\
& +\frac{a(a+1)(a+2)(\ell-1)}{2 \ell-1} \sin x \cos ^{2} x \cos 3 x+\cdots \\
& +\frac{a(a+1)(k-1)}{2 k-1} \sin ^{2} x \sin 2 x \\
& +\frac{a(a+1)(a+2)(k-1)}{2 k-1} \sin ^{2} x \cos x \sin 3 x+\cdots \\
& -\frac{a(a+1)(a+2)(k-2)}{3(2 k-1)} \sin ^{3} x \cos 3 x-\cdots \\
& -\cdots \text {. }
\end{aligned}
$$

Evidently, significant simplification in the double sums $P_{k, \ell}(x), Q_{k, \ell}(x)$ is possible if we collect terms to the same Pochhammer products $(a)_{p+q}$. If both $k \geq 3$ and $\ell \geq 3$, we have

$$
\begin{aligned}
P_{k, \ell}(x)= & 1+a+\frac{a(a+1)}{2}\left(1-B_{k, \ell}(x) \cos 2 x\right) \\
& +\frac{a(a+1)(a+2)}{6}\left(1-3 B_{k, \ell}(x) \cos 2 x\right)+\cdots, \\
Q_{k, \ell}(x)= & \frac{a(a+1)}{2} B_{k, \ell}(x) \sin 2 x+\frac{a(a+1)(a+2)}{2} B_{k, \ell}(x) \sin 2 x+\cdots,
\end{aligned}
$$

where

$$
B_{k, \ell}(x)=\frac{\cos ^{2} x}{2 \ell-1}-\frac{\sin ^{2} x}{2 k-1} .
$$

Simplification in the symmetric case $k=\ell$ is particularly significant, as could be expected from relation (4.8) for the case $\ell=0$. Quadratic transformation (2.4) can be written as

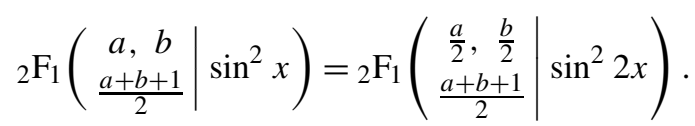


This relates the case $\ell=k$ with $a \mapsto 2 a$ of (6.6) to the case $\ell=0$ with $x \rightarrow 2 x$ of (6.6). To use (6.11) similarly with (6.7), one has to apply Euler's transformation (2.2) to the ${ }_{2} \mathrm{~F}_{1}$ function in (6.7) first.

For the dihedral functions with small $k, \ell$ nice expressions are obtained if we make the substitution $a \mapsto \lambda-k-\ell$ and write the right-hand sides of (6.6) and (6.7) as, respectively,

$$
\begin{aligned}
& \mathbf{P}_{k, \ell}(x) \cos \lambda x+\mathbf{Q}_{k, \ell}(x) \sin \lambda x, \\
& \mathbf{P}_{k, \ell}(x) \sin \lambda x-\mathbf{Q}_{k, \ell}(x) \cos \lambda x .
\end{aligned}
$$

Explicitly, we have the rotation transformation

$$
\begin{aligned}
& \mathbf{P}_{k, \ell}(x)=P_{k, \ell}(x) \cos (k+\ell) x-Q_{k, \ell} \sin (k+\ell) x, \\
& \mathbf{Q}_{k, \ell}(x)=P_{k, \ell}(x) \sin (k+\ell) x+Q_{k, \ell} \cos (k+\ell) x .
\end{aligned}
$$

A bit of a tedious translation of (6.10) shows the following effect of interchanging the indices $k, \ell$ :

$$
\begin{gathered}
\mathbf{P}_{\ell, k}(x)= \begin{cases}(-1)^{j} \mathbf{P}_{k, \ell}\left(\frac{\pi}{2}-x\right), & \text { if } k+\ell=2 j \text { even, } \\
(-1)^{j} \mathbf{Q}_{k, \ell}\left(\frac{\pi}{2}-x\right), & \text { if } k+\ell=2 j+1 \text { odd },\end{cases} \\
\mathbf{Q}_{\ell, k}(x)= \begin{cases}(-1)^{j-1} \mathbf{Q}_{k, \ell}\left(\frac{\pi}{2}-x\right), & \text { if } k+\ell=2 j \text { even }, \\
(-1)^{j} \mathbf{P}_{k, \ell}\left(\frac{\pi}{2}-x\right), & \text { if } k+\ell=2 j+1 \text { odd } .\end{cases}
\end{gathered}
$$

But here are attractive evaluation formulas after the substitution $a \mapsto \lambda-k-\ell$ :

$$
\begin{aligned}
{ }_{2} \mathrm{~F}_{1}\left(\begin{array}{c}
\frac{-1+\lambda}{2}, \frac{-1-\lambda}{2} \mid \\
\frac{1}{2}
\end{array} \mid \sin ^{2} x\right) & =\cos x \cos \lambda x+\sin x \frac{\sin \lambda x}{\lambda}, \\
{ }_{2} \mathrm{~F}_{1}\left(\begin{array}{c}
\frac{-1+\lambda}{2}, \frac{-1-\lambda}{2} \mid \\
-\frac{1}{2}
\end{array} \mid \sin ^{2} x\right) & =\cos x \cos \lambda x+\lambda \sin x \sin \lambda x, \\
{ }_{2} \mathrm{~F}_{1}\left(\begin{array}{c}
\frac{\lambda}{2},-\frac{\lambda}{2} \\
\frac{3}{2}
\end{array} \mid \sin ^{2} x\right) & =\frac{\lambda \cos x \sin \lambda x-\sin x \cos \lambda x}{\left(\lambda^{2}-1\right) \sin x}, \\
{ }_{2} \mathrm{~F}_{1}\left(\begin{array}{c}
\frac{2+\lambda}{2}, \frac{2-\lambda}{2} \mid \\
\frac{5}{2}
\end{array} \mid \sin ^{2} x\right) & =3 \frac{\cos x \sin \lambda x-\lambda \sin x \cos \lambda x}{\lambda(\lambda-1)(\lambda+1) \sin 3 x}, \\
{ }_{2} \mathrm{~F}_{1}\left(\begin{array}{c}
\frac{-2+\lambda}{2}, \frac{-2-\lambda}{2} \mid \\
-\frac{1}{2}
\end{array} \mid \sin ^{2} x\right) & =\cos 2 x \cos \lambda x+\frac{\lambda}{2} \sin 2 x \sin \lambda x, \\
{ }_{2} \mathrm{~F}_{1}\left(\begin{array}{c}
\frac{3+\lambda}{2}, \frac{3-\lambda}{2} \mid \\
\frac{5}{2}
\end{array} \mid \sin ^{2} x\right) & =3 \frac{\cos 2 x \sin \lambda x-\frac{1}{2} \lambda \sin 2 x \cos \lambda x}{\lambda(\lambda-2)(\lambda+2) \sin ^{3} x} .
\end{aligned}
$$


Some further expressions are

$$
\begin{aligned}
& \mathbf{P}_{0,2}(x)=\frac{\lambda^{2} \cos ^{2} x-2 \sin ^{2} x-1}{3}, \quad \mathbf{Q}_{0,2}(x)=\frac{\lambda \sin 2 x}{2}, \\
& \mathbf{P}_{1,2}(x)=\frac{\lambda(\lambda-2)}{3} \cos x(2 \cos 2 x-1), \\
& \mathbf{Q}_{1,2}(x)=\frac{\lambda-2}{3} \sin x\left(\lambda^{2} \cos ^{2} x-3 \sin ^{2} x\right), \\
& \mathbf{P}_{2,2}(x)=\frac{(\lambda-1)(\lambda-3)}{9}\left(\cos 4 x+2-\frac{\lambda^{2} \sin ^{2} 2 x}{4}\right), \\
& \mathbf{Q}_{2,2}(x)=\frac{\lambda(\lambda-1)(\lambda-3)}{12} \sin 4 x, \\
& \mathbf{P}_{0,3}(x)=\frac{\lambda \cos x\left(\lambda^{2} \cos ^{2} x-11 \sin ^{2} x-4\right)}{15}, \\
& \mathbf{Q}_{0,3}(x)=\frac{\sin x\left(2 \lambda^{2} \sin ^{2} x-2 \sin ^{2} x-3\right)}{5}, \\
& \mathbf{P}_{1,3}(x)=\frac{\lambda-3}{15}\left(\lambda^{2} \cos ^{2} x\left(1-7 \sin ^{2} x\right)+\cos 4 x+16 \sin ^{2} x-2\right), \\
& \mathbf{Q}_{1,3}(x)=\frac{\lambda(\lambda-3)}{30} \sin 2 x\left(\lambda^{2} \cos ^{2} x-14 \sin ^{2} x-1\right), \\
& \mathbf{P}_{3,3}(x)=\frac{(\lambda-1)(\lambda-3)(\lambda-5)}{75} \cos 2 x\left(\cos 4 x+4-\frac{\lambda^{2} \sin ^{2} 2 x}{2}\right), \\
& \mathbf{Q}_{3,3}(x)=\frac{\lambda(\lambda-1)(\lambda-3)(\lambda-5)}{900} \sin 2 x\left(11 \cos 4 x+19-\frac{\lambda^{2} \sin ^{2} 2 x}{2}\right), \\
& \mathbf{P}_{0,4}(x)=\frac{\lambda^{4} \cos ^{4} x}{105}-\frac{\lambda^{2} \cos ^{2} x\left(7 \sin ^{2} x+2\right)}{21}+\frac{\cos 4 x+32 \sin ^{2} x+2}{35}, \\
& \mathbf{Q}_{0,4}(x)=\frac{\lambda \sin 2 x\left(2 \lambda^{2} \cos ^{2} x-10 \sin ^{2} x-11\right)}{42} .
\end{aligned}
$$

\subsection{Apparent Padé approximation properties}

Because of the $O\left(x^{2 k+1}\right)$ solution in (6.7), the introduced trigonometric polynomials have properties resembling simultaneous Padé approximation. We have

$$
\frac{Q_{k, \ell}(x)}{P_{k, \ell}(x)}=\tan a x+O\left(x^{2 k+1}\right), \quad \frac{\mathbf{Q}_{k, \ell}(x)}{\mathbf{P}_{k, \ell}(x)}=\tan \lambda x+O\left(x^{2 k+1}\right) .
$$

and by the symmetry (6.10) or (6.15) and (6.16) of $k, \ell$,

$$
\frac{Q_{k, \ell}\left(\frac{\pi}{2}-x\right)}{P_{k, \ell}\left(\frac{\pi}{2}-x\right)}=-\tan a x+O\left(x^{2 \ell+1}\right), \quad\left(\frac{\mathbf{P}_{k, \ell}\left(\frac{\pi}{2}-x\right)}{\mathbf{Q}_{k, \ell}\left(\frac{\pi}{2}-x\right)}\right)^{\varepsilon}=\varepsilon \tan \lambda x+O\left(x^{2 \ell+1}\right) .
$$

Here $\varepsilon=(-1)^{k+\ell-1}$. It is however not clear what characterizes the trigonometric polynomials uniquely in the approximation context. From the degenerate cases with vanishing Pochhammer factors on the right-hand sides of (6.6) or (6.7) we get the following 
vanishing or exact approximation properties:

$$
\begin{array}{r}
P_{k, \ell}(x)=0, \quad Q_{k, \ell}(x)=0, \quad \text { if (5.6) holds, } \\
\frac{Q_{k, \ell}(x)}{P_{k, \ell}(x)}=\tan a x, \quad \text { if (5.8) holds, } \\
\frac{P_{k, \ell}(x)}{Q_{k, \ell}(x)}=-\tan a x, \quad \text { if (5.10) holds, } \\
\mathbf{P}_{k, \ell}(x)=0, \quad \mathbf{Q}_{k, \ell}(x)=0, \quad \text { if (5.7) holds, } \\
\frac{\mathbf{Q}_{k, \ell}(x)}{\mathbf{P}_{k, \ell}(x)}=\tan \lambda x, \quad \text { if (5.9) holds, } \\
\frac{\mathbf{P}_{k, \ell}(x)}{\mathbf{Q}_{k, \ell}(x)}=-\tan \lambda x,
\end{array}
$$

and similarly with $P_{k, \ell}(\pi / 2-x), Q_{k, \ell}(\pi / 2-x)$ or $\mathbf{P}_{k, \ell}(\pi / 2-x), \mathbf{Q}_{k, \ell}(\pi / 2-x)$. These conditions do not determine the trigonometric polynomials uniquely either, because $\mathbf{P}_{k, \ell}(x), \mathbf{Q}_{k, \ell}(x)$ are not the shifted $a \mapsto \lambda-k-\ell$ versions of $P_{k, \ell}(x), Q_{k, \ell}(x)$.

\subsection{Contiguous relations}

Contiguous relations for the considered ${ }_{2} \mathrm{~F}_{1}$ functions translate into the following recurrence relations:

$$
\begin{aligned}
P_{k, \ell}(x) & =\frac{a+2 k-1}{2 k-1} \sin ^{2} x P_{k-1, \ell}(x)+\frac{a+2 \ell-1}{2 \ell-1} \cos ^{2} x P_{k, \ell-1}(x) \\
\left(\frac{a}{2}+k+\ell+1\right) P_{k, \ell}(x)= & \left(k+\frac{1}{2}\right) P_{k+1, \ell}(x)+\left(\ell+\frac{1}{2}\right) P_{k, \ell+1}(x) \\
\left(\ell+\frac{1}{2}\right) P_{k, \ell+1}(x)= & \left(\ell+\frac{1}{2}+(a+k+\ell) \cos ^{2} x\right) P_{k, \ell}(x) \\
& -\frac{a+2 \ell-1}{2 \ell-1}\left(\frac{a}{2}+k+\ell\right) \cos ^{2} x P_{k, \ell-1}(x)
\end{aligned}
$$

The recurrence relations for the $Q_{k, \ell}$ are the same. Three-term recurrences for the trigonometric polynomials $\mathbf{P}_{k, \ell}(x), \mathbf{Q}_{k, \ell}(x)$ are less clear, because some shifts in $k, \ell$ lead to half-integer shifts in the upper parameters of the ${ }_{2} \mathrm{~F}_{1}$ functions, and transformation (6.14) mixes the $\mathbf{P}_{k, \ell}$ with the $\mathbf{Q}_{k, \ell}$. Since we formally have $\mathbf{Q}_{0,0}(x)=0, \mathbf{Q}_{0,-1}(x)=0$, three-term recurrences between these polynomials with minimal shifts in $k, \ell$ should not be expected. Here are some consequences of the contiguous relations, nevertheless:

$$
\begin{aligned}
\left(2 k \cos ^{2} x+2 \ell \sin ^{2} x+1\right) \mathbf{P}_{k, \ell}(x)= & \frac{2 k+1}{2 \ell-1}(\lambda-k+\ell-1) \cos ^{2} x \mathbf{P}_{k+1, \ell-1}(x) \\
& +\frac{2 \ell+1}{2 k-1}(\lambda+k-\ell-1) \sin ^{2} x \mathbf{P}_{k-1, \ell+1}(x),
\end{aligned}
$$


166

$$
\begin{aligned}
\frac{\sin ^{2} x}{2 k+1} \mathbf{P}_{k, \ell}(x)+\frac{\mathbf{P}_{k+1, \ell+1}(x)}{\lambda-k-\ell-1}= & \frac{\lambda-k+\ell-1}{(2 \ell-1)(2 \ell+1)} \cos ^{2} x \mathbf{P}_{k+1, \ell-1}(x) \\
(2 \ell-1) \mathbf{P}_{k, \ell}(x)= & \frac{\lambda^{2}-(k+\ell)^{2}}{(2 k-1)(2 k+1)}(\lambda-k-\ell+1) \sin ^{2} x \mathbf{P}_{k-1, \ell-1}(x) \\
& +(\lambda-k+\ell-1) \mathbf{P}_{k+1, \ell-1}(x) .
\end{aligned}
$$

\subsection{A hyperbolic trigonometric version of (3.1)}

A trigonometric version of formula (3.1) is pointless, because the substitution $z \mapsto-\tan ^{2} x$ does not give a meaningful ${ }_{2} \mathrm{~F}_{1}$ argument for a local expansion. We may, however, use the hyperbolic tangent substitution $z \mapsto \tanh ^{2} x$ (plus $a \mapsto \lambda-k-\ell$ for a little neatness), and consider the approximation $x \rightarrow+\infty$ :

$$
\begin{aligned}
&{ }_{2} \mathrm{~F}_{1}\left(\begin{array}{c|c}
\frac{\lambda-k-\ell}{2}, \frac{\lambda-k+\ell+1}{2} & \frac{1}{\cosh ^{2} x}
\end{array}\right) \\
&=2^{\lambda} \cosh ^{\lambda} x(\cosh \lambda x-\sinh \lambda x) \tanh ^{k} x \\
& \quad \times F_{3}\left(\begin{array}{c}
k+1, \ell+1 ;-k,-\ell \\
\lambda+1
\end{array}\right.\left.\frac{1-\operatorname{coth} x}{2}, \frac{1-\tanh x}{2}\right) .
\end{aligned}
$$

Trigonometric expressions for the dihedral functions with $k \ell=0$ or $k=\ell$ could be obtained in relation to the Legendre functions (as described in Remark 4.2). Infinite trigonometric series for general Legendre functions are given in [1, 8.7.1-8.7.2].

\subsection{Trigonometric expressions for logarithmic solutions}

The use of trigonometric arguments is instructive for degenerate or logarithmic dihedral ${ }_{2} \mathrm{~F}_{1}$ functions as well, as suggested by formulas (1.4) and (1.5). In the logarithmic cases (5.8) and (5.10) the non-logarithmic solution like (5.14) in (3.2) or (3.3) is, respectively,

$$
\frac{P_{k, \ell}(x)}{\cos a x}, \quad-\frac{Q_{k, \ell}(x)}{\cos a x},
$$

due to (6.25). The logarithmic solution is obtained by differentiating (6.9) or (6.8) with respect to $a$. Under condition (5.8), the left-hand side of (5.19) if $a$ is even, or (5.20) if $a$ is odd, is equal to

$$
\frac{x P_{k, \ell}(x)}{\cos a x}+\frac{d P_{k, \ell}(x)}{d a} \sin a x-\frac{d Q_{k, \ell}(x)}{d a} \cos a x, \quad \text { with } a=-m .
$$

Under condition (5.10), the left-hand side of (5.21) is equal to

$$
\frac{x Q_{k, \ell}(x)}{\cos a x}+\frac{d P_{k, \ell}(x)}{d a} \cos a x+\frac{d Q_{k, \ell}(x)}{d a} \sin a x, \quad \text { with } a=-m .
$$

Similar expressions can be obtained with the more compact trigonometric polynomials $\mathbf{P}_{k, \ell}(x), \mathbf{Q}_{k, \ell}(x)$, which depend on the parameter $\lambda=a+k+\ell$. 
Acknowledgement. The author was supported by the JSPS grant No 20740075 .

\title{
REFERENCES
}

[1] M. Abramowitz and I. A. Stegun. Handbook of Mathematical Functions, with Formulas, Graphs and Mathematical Tables (Applied Mathematics Series, 55). National Bureau of Standards, Washington, D.C., 1964.

[2] G. E. Andrews, R. Askey and R. Roy. Special Functions. Cambridge University Press, Cambridge, 1999.

[3] F. Beukers. Gauss' hypergeometric function. The Mathematical Legacy of Wilhelm Magnus: Groups, Geometry and Special Functions (Contemporary Mathematics Series, 169). Eds W. Abikoff et al. American Mathematical Society, Providence, 2007, pp. 29-43.

[4] A. Erdélyi. Higher Transcendental Functions. Vol. I. McGraw-Hill Book Company, New York, 1953.

[5] M. Petkovšek, H. S. Wilf and D. Zeilberger. $A=B$. A K Peters Ltd, Wellesley, MA, 1996.

[6] H. M. Srivastava and P. W. Karlsson. Multiple Gaussian Hypergeometric Series. Ellis Horwood Ltd., 1985.

[7] R. Vidunas. Degenerate Gauss hypergeometric functions. Kyushu J. Math. 61 (2007), 109-135.

[8] R. Vidunas. Transformations and invariants for dihedral Gauss hypergeometric functions. Available at http://arxiv.org/abs/1101.3688.

[9] R. Vidunas. Specialization of Appell's functions to univariate hypergeometric functions. J. Math. Anal. Appl. 355 (2009), 145-163.

[10] Wikipedia. Hypergeometric series. http://en.wikipedia.org/wiki/Hypergeometric_series.

\author{
Raimundas Vidunas \\ Kobe University \\ Organization for Advanced Science and Technology (OAST) \\ Rokko-dai 1-1, Nada-ku \\ Kobe 657-8501 \\ Japan \\ (E-mail: vidunas@math.kobe-u.ac.jp)
}

